Pacific

Journal of

Mathematics

A CERTAIN QUOTIENT OF ETA-FUNCTIONS FOUND IN RAMANUJAN'S LOST NOTEBOOK

Bruce C. Berndt, Heng Huat Chan, Soon-Yi Kang,

AND LIANG-ChENG ZHANG 


\title{
A CERTAIN QUOTIENT OF ETA-FUNCTIONS FOUND IN RAMANUJAN'S LOST NOTEBOOK
}

\author{
Bruce C. Berndt, Heng Huat Chan, Soon-Yi Kang, \\ AND LiAng-Cheng ZHANG
}

In his lost notebook, Ramanujan defined a parameter $\lambda_{n}$ by a certain quotient of Dedekind eta-functions at the argument $q=\exp (-\pi \sqrt{n / 3})$. He then recorded a table of several values of $\lambda_{n}$. To prove these values (and others), we develop several methods, which include modular equations, the modular $j$-invariant, Kronecker's limit formula, Ramanujan's "cubic theory" of elliptic functions, and an empirical process.

\section{Introduction.}

On the top of the page 212 in his lost notebook, Ramanujan defined the function $\lambda_{n}$ by

$$
\lambda_{n}=\frac{e^{\pi / 2 \sqrt{n / 3}}}{3 \sqrt{3}}\left\{\left(1+e^{-\pi \sqrt{n / 3}}\right)\left(1-e^{-2 \pi \sqrt{n / 3}}\right)\left(1-e^{-4 \pi \sqrt{n / 3}}\right) \cdots\right\}^{6},
$$

and then devoted the remainder of the page to stating several elegant values of $\lambda_{n}$, for $n \equiv 1(\bmod 8)$, namely:

$$
\begin{aligned}
& \lambda_{1}=1, \quad \lambda_{9}=3, \quad \lambda_{17}=4+\sqrt{17}, \quad \lambda_{25}=(2+\sqrt{5})^{2}, \\
& \lambda_{33}=18+3 \sqrt{33}, \quad \lambda_{41}=32+5 \sqrt{41}, \quad \lambda_{49}=55+12 \sqrt{21}, \\
& \lambda_{57}=, \quad \lambda_{65}=, \quad \lambda_{81}=, \quad \lambda_{89}=500+53 \sqrt{89}, \\
& \lambda_{73}=\left(\sqrt{\frac{11+\sqrt{73}}{8}}+\sqrt{\frac{3+\sqrt{73}}{8}}\right)^{6}, \\
& \lambda_{97}=\left(\sqrt{\frac{17+\sqrt{97}}{8}}+\sqrt{\frac{9+\sqrt{97}}{8}}\right)^{6}, \\
& \lambda_{121}=\left(\frac{3 \sqrt{3}+\sqrt{11}}{4}+\sqrt{\frac{11+3 \sqrt{33}}{8}}\right)^{6}, \\
& \lambda_{169}=, \quad \lambda_{193}=, \quad \lambda_{217}=, \quad \lambda_{241}=,
\end{aligned}
$$




$$
\lambda_{265}=, \quad \lambda_{289}=, \quad \lambda_{361}=.
$$

Note that for several values of $n$, Ramanujan did not record the corresponding value of $\lambda_{n}$.

The purpose of this paper is to establish all the values of $\lambda_{n}$ in (1.2), including the ones that are not explicitly stated by Ramanujan, by using the modular $j$-invariant, modular equations, Kronecker's limit formula, and an empirical approach. Applications of values for $\lambda_{n}$ will appear in papers by Chan, W.-C. Liaw, and V. Tan [16], Chan, A. Gee, and Tan [14], and by Berndt and Chan [6].

The function $\lambda_{n}$ had been briefly introduced earlier in his third notebook [25, p. 393], where Ramanujan offered a formula for $\lambda_{n}$ in terms of Klein's $j$-invariant, first proved by Berndt and Chan [5], [3, p. 318, Entry 11.21] by using Ramanujan's cubic theory of elliptic functions to alternative bases. As K.G. Ramanathan [23] pointed out, the formula in the third notebook is for evaluating $\lambda_{n / 3}$, especially for $n=11,19,43,67,163$. Observe that $-11,-19,-43,-67$, and -163 are precisely the discriminants congruent to 5 modulo 8 of imaginary quadratic fields of class number one. (Ramanathan inadvertently inverted the roles of $n$ and $n / 3$ in his corresponding remark.) In Section 2, we discuss some of these results in Ramanujan's third notebook and show how they can be used to calculate the values of $\lambda_{n}$ when $3 \mid n$.

In this and the next two paragraphs, we offer some necessary definitions. Let $\eta(\tau)$ denote the Dedekind eta-function, defined by

$$
\eta(\tau):=e^{2 \pi i \tau / 24} \prod_{n=1}^{\infty}\left(1-e^{2 \pi i n \tau}\right)=: q^{1 / 24} f(-q),
$$

where $q=e^{2 \pi i \tau}$ and $\operatorname{Im} \tau>0$. Then (1.1) can be written in the alternative form

$$
\lambda_{n}=\frac{1}{3 \sqrt{3}} \frac{f^{6}(q)}{\sqrt{q} f^{6}\left(q^{3}\right)}=\frac{1}{3 \sqrt{3}}\left(\frac{\eta\left(\frac{1+i \sqrt{n / 3}}{2}\right)}{\eta\left(\frac{1+i \sqrt{3 n}}{2}\right)}\right)^{6},
$$

where $q=e^{-\pi \sqrt{n / 3}}$.

Since much of this paper is devoted to the evaluation of $\lambda_{n}$ by using modular equations, we now give a definition of a modular equation. Let $(a)_{k}=(a)(a+1) \cdots(a+k-1)$ and define the ordinary hypergeometric function ${ }_{2} F_{1}(a, b ; c ; z)$ by

$$
{ }_{2} F_{1}(a, b ; c ; z):=\sum_{k=0}^{\infty} \frac{(a)_{k}(b)_{k}}{(c)_{k}} \frac{z^{k}}{k !}, \quad|z|<1 .
$$


Suppose that

$$
\frac{{ }_{2} F_{1}\left(\frac{1}{2}, \frac{1}{2} ; 1 ; 1-\beta\right)}{{ }_{2} F_{1}\left(\frac{1}{2}, \frac{1}{2} ; 1 ; \beta\right)}=n \frac{{ }_{2} F_{1}\left(\frac{1}{2}, \frac{1}{2} ; 1 ; 1-\alpha\right)}{{ }_{2} F_{1}\left(\frac{1}{2}, \frac{1}{2} ; 1 ; \alpha\right)},
$$

for some positive integer $n$. A relation between the moduli $\sqrt{\alpha}$ and $\sqrt{\beta}$ induced by (1.6) is called a modular equation of degree $n$, and $\beta$ is said to have degree $n$ over $\alpha$.

In Section 3, using a modular equation of degree 3, we derive a formula for $\lambda_{n}$ in terms of the Ramanujan-Weber class invariant which is defined by

$$
G_{n}:=2^{-1 / 4} q^{-1 / 24}\left(-q ; q^{2}\right)_{\infty},
$$

where $q=\exp (-\pi \sqrt{n})$ and

$$
(a ; q)_{\infty}=\prod_{n=0}^{\infty}\left(1-a q^{n}\right) .
$$

In Sections 4 and 5, we establish all 8 values of $\lambda_{p^{2}}$ in the table (1.2). Our proofs in Section 4 employ certain modular equations of degrees 3, 5, 7, and 11. The first three were claimed by Ramanujan [25], and the last one was newly discovered by Berndt, S. Bhargava, and F. G. Garvan [4] as a modular equation in the theory of signature 3 in [4]. In the theory of signature 3 , we say that the modulus $\sqrt{\beta}$ has degree $n$ over the modulus $\sqrt{\alpha}$ when

$$
\frac{{ }_{2} F_{1}\left(\frac{1}{3}, \frac{2}{3} ; 1 ; 1-\beta\right)}{{ }_{2} F_{1}\left(\frac{1}{3}, \frac{2}{3} ; 1 ; \beta\right)}=n \frac{{ }_{2} F_{1}\left(\frac{1}{3}, \frac{2}{3} ; 1 ; 1-\alpha\right)}{{ }_{2} F_{1}\left(\frac{1}{3}, \frac{2}{3} ; 1 ; \alpha\right)} .
$$

A modular equation of degree $n$ in the theory of signature 3 is a relation between $\alpha$ and $\beta$ which is induced by (1.8). In Section 5, we employ recent discoveries of Chan and W.-C. Liaw [15], [20] on Russell-type modular equations of degrees 13, 17, and 19 in the theory of signature 3 . In these two sections, we also determine the values of $\lambda_{5}, \lambda_{7}, \lambda_{11}$, and $\lambda_{17}$.

In two papers [22], [23], using Kronecker's limit formula, Ramanathan determined several values of $\lambda_{n}$. In [23], in order to determine two specific values of the Rogers-Ramanujan continued fraction, he evaluated $\lambda_{25}$ by applying Kronecker's limit formula to $L$-functions of orders of $\mathbb{Q}(\sqrt{-3})$ with conductor 5 . This method was also used to determine $\lambda_{49}$. In the other paper [22], Ramanathan found a representation for $\lambda_{n}$ in terms of fundamental units, where $-3 n$ is a fundamental discriminant of an imaginary quadratic field $\mathbb{Q}(\sqrt{-3 n})$ which has only one class in each genus of ideal classes. In particular, he calculated $\lambda_{17}, \lambda_{41}, \lambda_{65}, \lambda_{89}$, and $\lambda_{265}$. This 
formula and all 14 values of such $\lambda_{n}$ 's are given in Section 6. In the same section, we extend Ramanathan's method to establish a similar result for $\lambda_{n}$ when $-3 n \equiv 3(\bmod 4)$ and there is precisely one class per genus in each imaginary quadratic field $\mathbb{Q}(\sqrt{-3 n})$.

Through Section 6, all values of $\lambda_{n}$ in (1.2) are calculated except for $n=73,97,193,217,241$. In Section 7, we employ an empirical process, analogous to that employed by G. N. Watson $[29],[30]$ in his calculations of class invariants, to determine $\lambda_{n}$ for these remaining values of $n$. This empirical method has been put on a firm foundation by Chan, A. Gee and V. Tan [14]. Their method works whenever $3 \nmid n, n$ is squarefree, and the class group of $\mathbb{Q}(\sqrt{-3 n})$ takes the form $\mathbb{Z}_{2} \oplus \mathbb{Z}_{2} \oplus \cdots \oplus \mathbb{Z}_{2 k}$, with $1 \leq k \leq 4$.

The first representation of $\lambda_{n}$ in (1.4) suggests connections between $\lambda_{n}$ and Ramanujan's alternative cubic theory. In fact, Berndt and Chan [5] have recently found such a relationship. In Section 8, applying one of their results, we establish an explicit formula for the $j$-invariant in terms of $\lambda_{n}$, and evaluate several values of the $j$-invariant. Values of the $j$-invariant play an important role in generating rapidly convergent series for $1 / \pi$. For example, using the value of $\lambda_{1105}$, Berndt and Chan [6] established a series for $1 / \pi$ which yields about 73 or 74 digits of $\pi$ per term. The previous record, which yields 50 digits per term, was given by the Borweins [9] in 1988.

The values of $\lambda_{n}$ are not only related to convergent series for $1 / \pi$ via the $j$-invariants. In fact, Chan, Liaw, and Tan generated [16] a new class of series for $1 / \pi$ depending on values of $\lambda_{n}$. Using the values of $\lambda_{n}$ proved in this paper, they derived many simple series for $1 / \pi$ which are analogues of Ramanujan's series "belonging to the theory of $q_{2}$ ". For example, he proved that

$$
\frac{4}{\pi \sqrt{3}}=\sum_{k=0}^{\infty}(5 k+1) \frac{\left(\frac{1}{3}\right)_{k}\left(\frac{2}{3}\right)_{k}\left(\frac{1}{2}\right)_{k}}{(1)_{k}^{3}}\left(-\frac{9}{16}\right)^{k}
$$

which follows from the value $\lambda_{9}=3$ and a certain Lambert-type series identity.

In the table (1.2), we observe that if $n$ is not divisible by 3 , then $\lambda_{n}$ is a unit. In fact, in the final section, we show that $\lambda_{n}$ is a unit when $n$ is odd and $3 \nmid n$.

We conclude the introduction by summarizing in a table the values of $n$ for which $\lambda_{n}$ is determined in this paper, and the sections where the values can be located. 


\begin{tabular}{|r|c||c|c||r|c|}
\hline$n$ & Section $(s)$ & $n$ & Section & $n$ & Section \\
\hline 1 & 2,3 & 33 & 2 & 89 & 6 \\
3 & 3 & 35 & 6 & 91 & 6 \\
5 & 4,6 & 41 & 6 & 97 & 7 \\
7 & 4,6 & 49 & 4 & 115 & 6 \\
9 & 2,4 & 55 & 6 & 119 & 6 \\
11 & 4,6 & 57 & 2 & 121 & 4 \\
17 & 5,6 & 59 & 6 & 129 & 2 \\
19 & 6 & 65 & 6 & 145 & 6 \\
25 & 4 & 73 & 7 & 161 & 6 \\
31 & 6 & 81 & 4 & 169 & 5 \\
\hline
\end{tabular}

\begin{tabular}{|c|c||r|c|}
\hline$n$ & Section & \multicolumn{1}{|c|}{$n$} & Section \\
\hline 185 & 6 & 455 & 6 \\
193 & 7 & 489 & 2 \\
201 & 2 & 665 & 6 \\
209 & 6 & 1001 & 6 \\
217 & 7 & 1105 & 6 \\
241 & 7 & $11 / 3$ & 2 \\
265 & 6 & $19 / 3$ & 2 \\
289 & 5 & $43 / 3$ & 2 \\
361 & 5 & $67 / 3$ & 2 \\
385 & 6 & $163 / 3$ & 2 \\
\hline
\end{tabular}

2. $\lambda_{n}$ and the modular $j$-invariant.

Recall that $[\mathbf{1 7}$, p. 81] the invariants $J(\tau)$ and $j(\tau)$, for $\tau \in \mathbb{H}:=\{\tau: \operatorname{Im} \tau>$ $0\}$, are defined by

$$
J(\tau)=\frac{g_{2}^{3}(\tau)}{\Delta(\tau)} \quad \text { and } \quad j(\tau)=1728 J(\tau),
$$

where

$$
\begin{gathered}
\Delta(\tau)=g_{2}^{3}(\tau)-27 g_{3}^{2}(\tau), \\
g_{2}(\tau)=60 \sum_{\substack{m, n=-\infty \\
(m, n) \neq(0,0)}}^{\infty}(m \tau+n)^{-4},
\end{gathered}
$$

and

$$
g_{3}(\tau)=140 \sum_{\substack{m, n=-\infty \\(m, n) \neq(0,0)}}^{\infty}(m \tau+n)^{-6} .
$$

Furthermore, the function $\gamma_{2}(\tau)$ is defined by [18, p. 249]

$$
\gamma_{2}(\tau)=\sqrt[3]{j(\tau)}
$$


where that branch which is real when $\tau$ is purely imaginary is chosen.

In his third notebook, at the top of page 392 in the pagination of [26], Ramanujan defines a function $J_{n}$ by

$$
J_{n}=-\frac{1}{32} \gamma_{2}\left(\frac{3+\sqrt{-n}}{2}\right)=-\frac{1}{32} \sqrt[3]{j\left(\frac{3+\sqrt{-n}}{2}\right)} .
$$

For 15 values of $n, n \equiv 3(\bmod 4)$, Ramanujan indicates the corresponding values for $J_{n}$. See [3, pp. 310-312] for proofs of these evaluations. In particular,

$$
\begin{gathered}
J_{3}=0, \quad J_{27}=5 \cdot 3^{1 / 3}, \quad J_{51}=3(\sqrt{17}+4)^{2 / 3} \frac{5+\sqrt{17}}{2}, \\
J_{75}=3 \cdot 5^{1 / 6} \frac{69+31 \sqrt{5}}{2}, \quad J_{99}=(23+4 \sqrt{33})^{2 / 3} \frac{77+15 \sqrt{33}}{2} .
\end{gathered}
$$

The first five values of $n$ for $\lambda_{n}$ in (1.2) are those for which $3 n=3,27,51,75$, 99; the corresponding values of $J_{n}$ are given in (2.5). Then on the next page, which is the last page of his third notebook, Ramanujan gives a formula leading to a representation of $\lambda_{n}$.

Theorem 2.1 (Ramanujan). For $q=\exp (-\pi \sqrt{n})$, define

$$
R:=R_{n}:=3^{1 / 4} q^{1 / 36} \frac{f(q)}{f\left(q^{1 / 3}\right)} .
$$

Then

$$
\frac{3 \sqrt{3}}{R_{n}^{6}}=\sqrt{8 J_{n}+3}+\sqrt{2 \sqrt{64 J_{n}^{2}-24 J_{n}+9}-8 J_{n}+6} .
$$

Theorem 2.1 was first proved in [3, p. 318, Entry 11.21]. Since $\lambda_{n}=R_{3 n}^{-6}$ by (1.4) and (2.6), (2.7) may be restated as

$$
3 \sqrt{3} \lambda_{n / 3}=\sqrt{8 J_{n}+3}+\sqrt{2 \sqrt{64 J_{n}^{2}-24 J_{n}+9}-8 J_{n}+6} .
$$

By substituting $J_{3}=0$ into (2.8), we determine the first value of $\lambda_{n}$ in (1.2), and we state it as a corollary.

\section{Corollary 2.2.}

$$
\lambda_{1}=1 \text {. }
$$

Unfortunately, it is not so easy to find other values of $\lambda_{n}$ from Theorem 2.1. We have to struggle with complicated radicals even when $n=9$ for which $\lambda_{9}=3$. It seems that Ramanujan used this formula to determine the values of $\lambda_{n / 3}$ for rational integral values of $J_{n}$ as given in the following table, which constitutes the first part of the last page of the third notebook. 
$(2.9)$

\begin{tabular}{|c|c|c|c|}
\hline$n$ & $J_{n}$ & $8 J_{n}+3$ & $64 J_{n}^{2}-24 J_{n}+9$ \\
\hline 11 & 1 & 11 & $49=7^{2}$ \\
\hline 19 & 3 & 27 & $513=27 \cdot 19$ \\
\hline 43 & 30 & $243=27 \cdot 3^{2}$ & $56,889=27 \cdot 43 \cdot 7^{2}$ \\
\hline 67 & 165 & $1323=27 \cdot 7^{2}$ & $1,738,449=27 \cdot 31^{2} \cdot 67$ \\
\hline 163 & 20010 & $160,083=27 \cdot 77^{2}$ & $25,625,126,169=27 \cdot 163 \cdot 2413^{2}$ \\
\hline
\end{tabular}

But we are going to use Ramanujan's discoveries recorded between the table (2.9) and Theorem 2.1 on the last page in his third notebook. Ramanujan first sets, for $q=\exp (-\pi \sqrt{n})$,

$$
t_{n}:=\sqrt{3} q^{1 / 18} \frac{f\left(q^{1 / 3}\right) f\left(q^{3}\right)}{f^{2}(q)}
$$

and

$$
u_{n}:=\frac{1}{3} \sqrt{1+\frac{8}{3} J_{n}}
$$

(To avoid a conflict of notation, we have replaced Ramanujan's second $t_{n}$ by $u_{n}$.) He then asserted that

$$
t_{n}=\left(2 \sqrt{64 J_{n}^{2}-24 J_{n}+9}-\left(16 J_{n}-3\right)\right)^{1 / 6}
$$

and listed very simple polynomials satisfied by $t_{n}$ and $u_{n}$. The definition of $u_{n}$ in (2.11) seems unmotivated, but by recalling from the proof of Theorem 2.1 in $[\mathbf{3}$, p. $321,(11.33)]$ that

$$
2 \sqrt{8 J_{n}+3}=\frac{f^{6}\left(q^{1 / 3}\right)}{q^{1 / 6} f^{6}(q)}-27 \sqrt{q} \frac{f^{6}\left(q^{3}\right)}{f^{6}(q)},
$$

we find that

$$
2 u_{n}=\frac{1}{3 \sqrt{3}} \frac{f^{6}\left(q^{1 / 3}\right)}{q^{1 / 6} f^{6}(q)}-3 \sqrt{3} \sqrt{q} \frac{f^{6}\left(q^{3}\right)}{f^{6}(q)} .
$$

We summarize these results in the following two corollaries.

\section{Corollary 2.3.}

$$
\lambda_{n / 3}-\lambda_{3 n}^{-1}=\frac{2 \sqrt{8 J_{n}+3}}{3 \sqrt{3}} .
$$

Proof. This is a restatement of either (2.13) or (2.14), with the definition of $\lambda_{n}$ in (1.4). 


\section{Corollary 2.4.}

$$
\frac{\lambda_{3 n}}{\lambda_{n / 3}}=2 \sqrt{64 J_{n}^{2}-24 J_{n}+9}+\left(16 J_{n}-3\right) .
$$

Proof. By (1.4) and (2.10), $t_{n}^{6}=27 \lambda_{n / 3} \lambda_{3 n}^{-1}$. We obtain the result at once from (2.12).

\section{Corollary 2.5.}

$$
\lambda_{9}=3 .
$$

Proof. Let $n=3$ in either Corollary 2.3 or Corollary 2.4. The result follows immediately from the fact that $J_{3}=0$ and $\lambda_{1}=1$.

\section{Corollary 2.6.}

$$
\begin{aligned}
\lambda_{11 / 3} & =\frac{2 \sqrt{3}+\sqrt{11}}{3 \sqrt{3}}, \\
\lambda_{33} & =(3 \sqrt{3})(2 \sqrt{3}+\sqrt{11})=18+3 \sqrt{33} .
\end{aligned}
$$

Proof. (i) is an immediate consequence of Theorem 2.1 with $n=11$, since $J_{11}=1$. Using (i) and either Corollary 2.3 or Corollary 2.4 when $n=11$, we obtain (ii).

\section{Corollary 2.7.}

$$
\begin{aligned}
\lambda_{19 / 3} & =3^{-3 / 4} \sqrt{2 \sqrt{19}-5 \sqrt{3}}(\sqrt{46+6 \sqrt{57}}+\sqrt{45+6 \sqrt{57}}), \\
\lambda_{57} & =3^{3 / 4} \sqrt{2 \sqrt{19}+5 \sqrt{3}}(\sqrt{46+6 \sqrt{57}}+\sqrt{45+6 \sqrt{57}}) .
\end{aligned}
$$

Proof. Using (2.8) with $n=19$, we find that

$$
\lambda_{19 / 3}=3^{-3 / 4}(\sqrt{3 \sqrt{3}}+\sqrt{2(\sqrt{19}-\sqrt{3})}) .
$$

Let us represent $x=\sqrt{3 \sqrt{3}}+\sqrt{2(\sqrt{19}-\sqrt{3})}$ as a product of units. If $t=\sqrt{2(\sqrt{19}-\sqrt{3})}$, then $(x-t)^{2}=3 \sqrt{3}$, or

$$
x^{2}-2 t x+2 \sqrt{19}-5 \sqrt{3}=0 .
$$

Let

$$
y=\frac{x}{\sqrt{2 \sqrt{19}-5 \sqrt{3}}} .
$$

Then (2.15) becomes

$$
x \sqrt{2 \sqrt{19}-5 \sqrt{3}}\left(y-\frac{2 t}{\sqrt{2 \sqrt{19}-5 \sqrt{3}}}+\frac{1}{y}\right)=0 .
$$


Hence, by applying the quadratic formula to

$$
y+\frac{1}{y}=\frac{2 \sqrt{2 \sqrt{19}-2 \sqrt{3}}}{\sqrt{2 \sqrt{19}-5 \sqrt{3}}}=2 \sqrt{46+6 \sqrt{57}},
$$

we find that

$$
y=\sqrt{46+6 \sqrt{57}}+\sqrt{45+6 \sqrt{57}}
$$

from which (i) follows. From (i) and either Corollary 2.3 or Corollary 2.4 with $n=19$, we deduce (ii).

By similar methods, we can derive the following results.

\section{Corollary 2.8.}

(i)

$\lambda_{43 / 3}=3^{-3 / 4} \sqrt{14 \sqrt{43}-53 \sqrt{3}}(\sqrt{4294+378 \sqrt{129}}+\sqrt{4293+378 \sqrt{129}})$,

(ii)

$$
\lambda_{129}=3^{3 / 4} \sqrt{14 \sqrt{43}+53 \sqrt{3}}(\sqrt{4294+378 \sqrt{129}}+\sqrt{4293+378 \sqrt{129}}) .
$$

\section{Corollary 2.9.}

(i) $\quad \lambda_{67 / 3}=3^{-3 / 4} \sqrt{62 \sqrt{67}-293 \sqrt{3}}$

$$
\cdot(\sqrt{129214+9114 \sqrt{201}}+\sqrt{129213+9114 \sqrt{201}})
$$

(ii) $\quad \lambda_{201}=3^{3 / 4} \sqrt{62 \sqrt{67}+293 \sqrt{3}}$

$$
\cdot(\sqrt{129214+9114 \sqrt{201}}+\sqrt{129213+9114 \sqrt{201}}) .
$$

\section{Corollary 2.10.}

(i)

$$
\begin{aligned}
& \lambda_{163 / 3}=3^{-3 / 4} \sqrt{4826 \sqrt{163}-35573 \sqrt{3}} \\
& \quad \cdot(\sqrt{1898210854+85840062 \sqrt{489}}+\sqrt{1898210853+85840062 \sqrt{489}}),
\end{aligned}
$$

$$
\begin{aligned}
\lambda_{489} & =3^{3 / 4} \sqrt{4826 \sqrt{163}+35573 \sqrt{3}} \\
& (\sqrt{1898210854+85840062 \sqrt{489}}+\sqrt{1898210853+85840062 \sqrt{489}}) .
\end{aligned}
$$




\section{3. $\lambda_{n}$ and the class invariant $G_{n}$.}

In [23], Ramanathan introduced a new function $\mu_{n}$, defined by

$$
\mu_{n}:=\frac{1}{3 \sqrt{3}}\left(\frac{\eta(i \sqrt{n / 3})}{\eta(i \sqrt{3 n})}\right)^{6}=\frac{1}{3 \sqrt{3}} \frac{f^{6}\left(-q^{2}\right)}{q f^{6}\left(-q^{6}\right)}, \quad q=e^{-\pi \sqrt{n / 3}} .
$$

Then by (1.4), (3.1), and Euler's pentagonal number theorem, $f(-q)=$ $(q ; q)_{\infty}$

$$
\frac{\lambda_{n}}{\mu_{n}}=q^{1 / 2}\left(\frac{f(q) f\left(-q^{6}\right)}{f\left(-q^{2}\right) f\left(q^{3}\right)}\right)^{6}=q^{1 / 2}\left(\frac{\left(-q ; q^{2}\right)_{\infty}}{\left(-q^{3} ; q^{6}\right)_{\infty}}\right)^{6} .
$$

Hence from (1.7), we deduce the following result:

\section{Theorem 3.1.}

$$
\frac{\lambda_{n}}{\mu_{n}}=\left(\frac{G_{n / 3}}{G_{3 n}}\right)^{6}
$$

Let

$$
P=\frac{f(-q)}{q^{1 / 12} f\left(-q^{3}\right)} \quad \text { and } \quad Q_{p}=\frac{f\left(-q^{p}\right)}{q^{p / 12} f\left(-q^{3 p}\right)} .
$$

Recall the modular equation [2, p. 204, Entry 51]

$$
\left(P Q_{2}\right)^{2}+\frac{9}{\left(P Q_{2}\right)^{2}}=\left(\frac{Q_{2}}{P}\right)^{6}+\left(\frac{P}{Q_{2}}\right)^{6} .
$$

By replacing $q$ by $-q$ in (3.3), we deduce from (1.4), (3.1), (3.2) and Theorem 3.1 that

$$
3\left(\lambda_{n} \mu_{n}\right)^{1 / 3}-3\left(\lambda_{n} \mu_{n}\right)^{-1 / 3}=\left(\frac{G_{3 n}}{G_{n / 3}}\right)^{6}-\left(\frac{G_{n / 3}}{G_{3 n}}\right)^{6} .
$$

Solving (3.4) for $\lambda_{n} \mu_{n}$, we find that

$$
\lambda_{n} \mu_{n}=\left(\frac{c+\sqrt{c^{2}+9}}{3}\right)^{3},
$$

where $2 c=\left(\frac{G_{3 n}}{G_{n / 3}}\right)^{6}-\left(\frac{G_{n / 3}}{G_{3 n}}\right)^{6}$. Hence from Theorem 3.1 and (3.5), we derive the following theorem.

\section{Theorem 3.2.}

$$
\lambda_{n}=\left[\left(\frac{G_{n / 3}}{G_{3 n}}\right) \sqrt{\frac{c+\sqrt{c^{2}+9}}{3}}\right]^{3},
$$

where $2 c=\left(\frac{G_{3 n}}{G_{n / 3}}\right)^{6}-\left(\frac{G_{n / 3}}{G_{3 n}}\right)^{6}$. 
We give another proof of Corollary 2.2.

\section{Corollary 3.3.}

$$
\begin{aligned}
& \lambda_{1}=1, \\
& \mu_{1}=1 .
\end{aligned}
$$

Proof. Since $G_{1 / n}=G_{n}[\mathbf{2 4}],\left(G_{1 / 3} / G_{3}\right)^{6}=1$. Substituting this value into Theorem 3.2, we have (i), and then using Theorem 3.1, we deduce (ii) at once.

\section{Corollary 3.4.}

$$
\lambda_{3}=3^{3 / 4} \frac{\sqrt{3}-1}{\sqrt{2}} .
$$

Proof. Let $n=3$ in Theorem 3.2 and use the values [3, p. 189],

$$
G_{1}=1 \quad \text { and } \quad G_{9}=\left(\frac{1+\sqrt{3}}{\sqrt{2}}\right)^{1 / 3} \text {. }
$$

\section{4. $\lambda_{n}$ and modular equations.}

We shall employ a certain type of modular equation of degree $p$ in $P$ and $Q_{p}$ (defined in (3.2)) to calculate several values of $\lambda_{n}$. First, recall the modular equation of degree 9 [1, p. 346, Entry 1(iv)],

$$
1+9 q \frac{f^{3}\left(-q^{9}\right)}{f^{3}(-q)}=\left(1+27 q \frac{f^{12}\left(-q^{3}\right)}{f^{12}(-q)}\right)^{1 / 3} .
$$

After replacing $q$ by $-q$ in both sides, we deduce the following result from the definition of $\lambda_{n}$ in (1.4).

Theorem 4.1.

$$
1-\frac{1}{\lambda_{n}^{2}}=\left(1-\sqrt{\frac{3}{\lambda_{n} \lambda_{9 n}}}\right)^{3}
$$

\section{Corollary 4.2.}

$$
\begin{gathered}
\lambda_{9}=3 \\
\lambda_{81}=3 \sqrt[3]{3}\left(52+36 \sqrt[3]{3}+25 \sqrt[3]{3^{2}}\right) .
\end{gathered}
$$

Proof. Let $n=1$ and $n=9$ in Theorem 4.1 to obtain (i) and (ii), respectively.

At the end of Section 1, we remarked that $\lambda_{n}$ is a unit for odd $n$ not divisible by 3 . With this as motivation, set

$$
\lambda_{n}=(\sqrt{a+1}+\sqrt{a})^{6},
$$


and let

$$
\Lambda=\lambda_{n}^{1 / 3}+\lambda_{n}^{-1 / 3}
$$

Then

$$
a=\frac{\Lambda-2}{4} .
$$

By determining $\Lambda$ in (4.3) and then using (4.4) and (4.2), we next use modular equations to prove all the evaluations of $\lambda_{p^{2}}$ given explicitly by Ramanujan in (1.2).

\section{Theorem 4.3.}

$$
\left(27 \lambda_{n} \lambda_{25 n}\right)^{1 / 3}+\left(\frac{27}{\lambda_{n} \lambda_{25 n}}\right)^{1 / 3}=\left(\frac{\lambda_{25 n}}{\lambda_{n}}\right)^{1 / 2}-\left(\frac{\lambda_{n}}{\lambda_{25 n}}\right)^{1 / 2}+5 .
$$

Proof. From [2, p. 221, Entry 62], we find that

$$
\left(P Q_{5}\right)^{2}+5+\frac{9}{\left(P Q_{5}\right)^{2}}=\left(\frac{Q_{5}}{P}\right)^{3}-\left(\frac{P}{Q_{5}}\right)^{3},
$$

where $P$ and $Q_{5}$ are defined by (3.2). We can deduce Theorem 4.3 from (4.5) and (1.4) immediately after replacing $q$ by $-q$.

\section{Corollary 4.4 .}

$$
\lambda_{25}=\left(\frac{1+\sqrt{5}}{2}\right)^{6}=(2+\sqrt{5})^{2} .
$$

Proof. For brevity, we set $\lambda=\lambda_{25}$ in the proof. Let $n=1$ in Theorem 4.3. Then we have

$$
3\left(\lambda^{1 / 3}+\lambda^{-1 / 3}\right)=\left(\lambda^{1 / 2}-\lambda^{-1 / 2}\right)+5 .
$$

Set $\Lambda=\lambda^{1 / 3}+\lambda^{-1 / 3}$. Since

$$
\lambda^{1 / 2}-\lambda^{-1 / 2}=\left(\lambda^{1 / 6}-\lambda^{-1 / 6}\right)\left(\lambda^{1 / 3}+\lambda^{-1 / 3}+1\right),
$$

(4.6) becomes

$$
3 \Lambda-5=(\Lambda-2)^{1 / 2}(\Lambda+1),
$$

which can be simplified, after squaring both sides, to

$$
(\Lambda-3)^{3}=0 .
$$

Thus $\Lambda=3$ and $a=1 / 4$ by (4.4). Hence from (4.2),

$$
\lambda_{25}=\left(\sqrt{\frac{5}{4}}+\sqrt{\frac{1}{4}}\right)^{6} \text {. }
$$




\section{Theorem 4.5.}

$$
\begin{aligned}
& \left(27 \lambda_{n} \lambda_{49 n}\right)^{1 / 2}+\left(\frac{27}{\lambda_{n} \lambda_{49 n}}\right)^{1 / 2} \\
& =\left(\frac{\lambda_{49 n}}{\lambda_{n}}\right)^{2 / 3}+7\left(\frac{\lambda_{49 n}}{\lambda_{n}}\right)^{1 / 3}-7\left(\frac{\lambda_{n}}{\lambda_{49 n}}\right)^{1 / 3}-\left(\frac{\lambda_{n}}{\lambda_{49 n}}\right)^{2 / 3} .
\end{aligned}
$$

Proof. By (3.2) and (1.4), this theorem can be deduced from [2, p. 236, Entry 69]

$$
\left(P Q_{7}\right)^{3}+\frac{27}{\left(P Q_{7}\right)^{3}}=\left(\frac{Q_{7}}{P}\right)^{4}-7\left(\frac{Q_{7}}{P}\right)^{2}+7\left(\frac{P}{Q_{7}}\right)^{2}-\left(\frac{P}{Q_{7}}\right)^{4},
$$

with $q$ replaced by $-q$.

\section{Corollary 4.6.}

$$
\lambda_{49}=\left(\frac{\sqrt{7}+\sqrt{3}}{2}\right)^{6}=55+12 \sqrt{21} .
$$

Proof. Let $n=1$ in Theorem 4.5 and set $\lambda=\lambda_{49}$. Then

$$
\begin{aligned}
3 \sqrt{3}\left(\lambda^{1 / 2}+\lambda^{-1 / 2}\right) & =\lambda^{2 / 3}+7 \lambda^{1 / 3}-7 \lambda^{-1 / 3}-\lambda^{-2 / 3} \\
& =\left(\lambda^{1 / 3}-\lambda^{-1 / 3}\right)\left(\lambda^{1 / 3}+\lambda^{-1 / 3}+7\right),
\end{aligned}
$$

which can be simplified to

$$
3 \sqrt{3}\left(\lambda^{1 / 3}+\lambda^{-1 / 3}-1\right)=\left(\lambda^{1 / 6}-\lambda^{-1 / 6}\right)\left(\lambda^{1 / 3}+\lambda^{-1 / 3}+7\right) .
$$

Letting $\Lambda=\lambda^{1 / 3}+\lambda^{-1 / 3}$ in (4.8), we have

$$
3 \sqrt{3}(\Lambda-1)=\sqrt{\Lambda-2}(\Lambda+7) .
$$

Squaring both sides of (4.9), we deduce that

$$
(\Lambda-5)^{3}(\Lambda+2)=0 .
$$

Hence $\Lambda=5$ and

$$
\lambda_{49}=\left(\sqrt{\frac{7}{4}}+\sqrt{\frac{3}{4}}\right)^{6},
$$

by (4.2) and (4.4). 


\section{Theorem 4.7.}

$$
\begin{aligned}
& 9 \sqrt{3}\left\{\left(\lambda_{n} \lambda_{121 n}\right)^{5 / 6}+\left(\lambda_{n} \lambda_{121 n}\right)^{-5 / 6}\right\}-99\left\{\left(\lambda_{n} \lambda_{121 n}\right)^{2 / 3}+\left(\lambda_{n} \lambda_{121 n}\right)^{-2 / 3}\right\} \\
& +198 \sqrt{3}\left\{\left(\lambda_{n} \lambda_{121 n}\right)^{1 / 2}+\left(\lambda_{n} \lambda_{121 n}\right)^{-1 / 2}\right\} \\
& -759\left\{\left(\lambda_{n} \lambda_{121 n}\right)^{1 / 3}+\left(\lambda_{n} \lambda_{121 n}\right)^{-1 / 3}\right\} \\
& +693 \sqrt{3}\left\{\left(\lambda_{n} \lambda_{121 n}\right)^{1 / 6}+\left(\lambda_{n} \lambda_{121 n}\right)^{-1 / 6}\right\}-1386 \\
& =\left(\frac{\lambda_{n}}{\lambda_{121 n}}\right)+\left(\frac{\lambda_{121 n}}{\lambda_{n}}\right) .
\end{aligned}
$$

Proof. A new modular equation of degree 11, which was not mentioned by Ramanujan, was proved by Berndt, Bhargava, and Garvan [4], and is given by

$$
\begin{aligned}
& \left(P Q_{11}\right)^{5}+\left(\frac{3}{P Q_{11}}\right)^{5}+11\left\{\left(P Q_{11}\right)^{4}+\left(\frac{3}{P Q_{11}}\right)^{4}\right\}+66\left\{\left(P Q_{11}\right)^{3}\right. \\
& \left.+\left(\frac{3}{P Q_{11}}\right)^{3}\right\}+253\left\{\left(P Q_{11}\right)^{2}+\left(\frac{3}{P Q_{11}}\right)^{2}\right\}+693\left\{\left(P Q_{11}\right)\right. \\
& \left.+\left(\frac{3}{P Q_{11}}\right)\right\}+1368=\left(\frac{P}{Q_{11}}\right)^{6}+\left(\frac{Q_{11}}{P}\right)^{6},
\end{aligned}
$$

where $P$ and $Q_{11}$ are defined by (3.2). Replacing $q$ by $-q$ in the equation above yields Theorem 4.7 .

\section{Corollary 4.8.}

$$
\begin{aligned}
\lambda_{121} & =\left(\sqrt{\frac{19+3 \sqrt{33}}{8}}+\sqrt{\frac{11+3 \sqrt{33}}{8}}\right)^{6} \\
& =\left(\frac{3 \sqrt{3}+\sqrt{11}}{4}+\sqrt{\frac{11+3 \sqrt{33}}{8}}\right)^{6} .
\end{aligned}
$$

Proof. Letting $n=1$ and $\Lambda=\lambda_{121}^{1 / 3}+\lambda_{121}^{-1 / 3}$ in Theorem 4.7, we deduce that (4.11) $9 \sqrt{3}\left(\Lambda^{2}-\Lambda-1\right)(\Lambda+2)^{1 / 2}-99\left(\Lambda^{2}-2\right)+198 \sqrt{3}(\Lambda-1)(\Lambda+2)^{1 / 2}$

$$
-759 \Lambda+693 \sqrt{3}(\Lambda+2)^{1 / 2}-1386=\Lambda\left(\Lambda^{2}-3\right) .
$$

Rearranging (4.11) to

$$
9 \sqrt{3}(\Lambda+2)^{1 / 2}\left(\Lambda^{2}+21 \Lambda+54\right)=\Lambda^{3}+99 \Lambda^{2}+756 \Lambda+1188,
$$


and then squaring both sides, we deduce the equation

$$
\left(\Lambda^{2}-15 \Lambda-18\right)^{3}=0 .
$$

Thus

$$
\Lambda=\frac{3(5+\sqrt{33})}{2} .
$$

We complete the proof by using (4.2) and (4.4).

We now establish $\lambda_{5}, \lambda_{7}$, and $\lambda_{11}$ as well by using Theorems 4.3, 4.5, 4.7, and the following lemma.

\section{Lemma 4.9.}

$$
\lambda_{1 / n}=\frac{1}{\lambda_{n}}
$$

Proof. Recall that

$$
\eta(\tau+1)=e^{\pi i / 12} \eta(\tau) \quad \text { and } \quad \eta\left(-\frac{1}{\tau}\right)=(-i)^{1 / 2} \tau^{1 / 2} \eta(\tau) .
$$

From these properties of the Dedekind eta-function or Entry 27(iv), Ch. 16 in $[\mathbf{1}$, p. 43$]$, we find that

$$
\eta^{6}\left(\frac{1+i / \sqrt{3 n}}{2}\right)=(3 n \sqrt{3 n}) \eta^{6}\left(\frac{1+i \sqrt{3 n}}{2}\right)
$$

and

$$
\eta^{6}\left(\frac{1+i \sqrt{3 / n}}{2}\right)=\left(\frac{n}{3} \sqrt{\frac{n}{3}}\right) \eta^{6}\left(\frac{1+i \sqrt{n / 3}}{2}\right) .
$$

Hence the lemma follows from (1.4).

Corollary 4.10.

$$
\lambda_{5}=\frac{1+\sqrt{5}}{2} .
$$

Proof. Letting $n=1 / 5$ in Theorem 4.3 and using Lemma 4.9, we have

$$
1=\lambda_{5}-\lambda_{5}^{-1} \text {. }
$$

Solving this equation for $\lambda_{5}$ completes the proof.

\section{Corollary 4.11.}

$$
\lambda_{7}=(2+\sqrt{3})^{3 / 2}\left(\frac{\sqrt{3}+\sqrt{7}}{2}\right)^{-3 / 2} .
$$


Proof. Letting $n=1 / 7$ in Theorem 4.5 yields, by Lemma 4.9,

$$
6 \sqrt{3}=\lambda_{7}^{4 / 3}+7 \lambda_{7}^{2 / 3}-7 \lambda_{7}^{-2 / 3}-\lambda_{7}^{-4 / 3} .
$$

If we set $x=\lambda_{7}^{2 / 3}-\lambda_{7}^{-2 / 3}$, then (4.13) is equivalent to

$$
6 \sqrt{3}=x \sqrt{x^{2}+4}+7 x
$$

which has the solution $x=-2 \sqrt{3}+\sqrt{21}$. Hence by the quadratic formula,

$$
\begin{aligned}
\lambda_{7}^{2 / 3} & =\frac{-2 \sqrt{3}+\sqrt{21}+\sqrt{37-12 \sqrt{7}}}{2}=\frac{-2 \sqrt{3}+\sqrt{21}+2 \sqrt{7}-3}{2} \\
& =(2+\sqrt{3})\left(\frac{\sqrt{7}-\sqrt{3}}{2}\right)=(2+\sqrt{3})\left(\frac{\sqrt{7}+\sqrt{3}}{2}\right)^{-1} .
\end{aligned}
$$

Then the value of $\lambda_{7}$ follows immediately.

\section{Corollary 4.12.}

$$
\lambda_{11}=(2 \sqrt{3}+\sqrt{11})^{3 / 2}(10+3 \sqrt{11})^{-1 / 2} .
$$

Proof. If $n=1 / 11$ in Theorem 4.7, then by Lemma 4.9,

$$
\lambda_{11}^{2}+\lambda_{11}^{-2}=2(900 \sqrt{3}-1551) .
$$

By the quadratic formula,

$$
\begin{aligned}
\lambda_{11}^{2} & =900 \sqrt{3}-1551+10 \sqrt{48356-27918 \sqrt{3}} \\
& =900 \sqrt{3}-1551+470 \sqrt{11}-270 \sqrt{33} \\
& =(90 \sqrt{3}+47 \sqrt{11})(10-3 \sqrt{11}) \\
& =(2 \sqrt{3}+\sqrt{11})^{3}(10+3 \sqrt{11})^{-1} .
\end{aligned}
$$

Taking the positive square root above, we obtain the value of $\lambda_{11}$.

5. $\lambda_{n}$ and modular equations in the theory of signature 3 .

Suppose $\beta$ has degree $p$ over $\alpha$ in the theory of signature 3 , and let

$$
z_{1}:={ }_{2} F_{1}\left(\frac{1}{3}, \frac{2}{3} ; 1 ; \alpha\right) \quad \text { and } \quad z_{p}:={ }_{2} F_{1}\left(\frac{1}{3}, \frac{2}{3} ; 1 ; \beta\right) .
$$

For $\omega=\exp (2 \pi i / 3)$, if the cubic theta functions are defined by

$$
\begin{gathered}
a(q):=\sum_{m, n=-\infty}^{\infty} q^{m^{2}+m n+n^{2}}, \\
b(q):=\sum_{m, n=-\infty}^{\infty} \omega^{m-n} q^{m^{2}+m n+n^{2}},
\end{gathered}
$$


and

$$
c(q):=\sum_{m, n=-\infty}^{\infty} q^{(m+1 / 3)^{2}+(m+1 / 3)(n+1 / 3)+(n+1 / 3)^{2}},
$$

then [4, Lemma 2.6 and Corollary 3.2]

$$
a(q)=z_{1}, \quad b(q)=(1-\alpha)^{1 / 3} z_{1}, \quad c(q)=\alpha^{1 / 3} z_{1},
$$

and

$$
a\left(q^{p}\right)=z_{p}, \quad b\left(q^{p}\right)=(1-\beta)^{1 / 3} z_{p}, \quad c\left(q^{p}\right)=\beta^{1 / 3} z_{p} .
$$

Thus it follows that

$$
x:=(\alpha \beta)^{1 / 6}=\left(\frac{c(q) c\left(q^{p}\right)}{a(q) a\left(q^{p}\right)}\right)^{1 / 2}
$$

and

$$
y:=\{(1-\alpha)(1-\beta)\}^{1 / 6}=\left(\frac{b(q) b\left(q^{p}\right)}{a(q) a\left(q^{p}\right)}\right)^{1 / 2} .
$$

But, since [4, Lemma 5.1]

$$
b(q)=\frac{f^{3}(-q)}{f\left(-q^{3}\right)},
$$

$$
c(q)=3 q^{1 / 3} \frac{f^{3}\left(-q^{3}\right)}{f(-q)},
$$

and $[\mathbf{1 0}]$

$$
a^{3}(q)=b^{3}(q)+c^{3}(q),
$$

we find that

$$
a(q)=\left\{\frac{f^{12}(-q)+27 q f^{12}\left(-q^{3}\right)}{f^{3}(-q) f^{3}\left(-q^{3}\right)}\right\}^{1 / 3} .
$$

(This was also proved by Ramanujan; see [1, p. 460, Entry 3(i)].) Hence, by (5.4)-(5.7) and (5.9),

$$
x=\frac{3 q^{(p+1) / 6} f^{2}\left(-q^{3}\right) f^{2}\left(-q^{3 p}\right)}{\left\{f^{12}(-q)+27 q f^{12}\left(-q^{3}\right)\right\}^{1 / 6}\left\{f^{12}\left(-q^{p}\right)+27 q^{p} f^{12}\left(-q^{3 p}\right)\right\}^{1 / 6}}
$$

and

$$
y=\frac{f^{2}(-q) f^{2}\left(-q^{p}\right)}{\left\{f^{12}(-q)+27 q f^{12}\left(-q^{3}\right)\right\}^{1 / 6}\left\{f^{12}\left(-q^{p}\right)+27 q^{p} f^{12}\left(-q^{3 p}\right)\right\}^{1 / 6}} .
$$

Let

$$
T:=T(q):=\frac{f(-q)}{3^{1 / 4} q^{1 / 12} f\left(-q^{3}\right)} \quad \text { and } \quad U_{p}:=U_{p}(q):=\frac{f\left(-q^{p}\right)}{3^{1 / 4} q^{p / 12} f\left(-q^{3 p}\right)} .
$$


Then from (5.10) and (5.11), we find that

$$
\frac{x}{y}=\left(T U_{p}\right)^{-2}
$$

and

$$
x y=\left(T^{6}+T^{-6}\right)^{-1 / 3}\left(U_{p}^{6}+U_{p}^{-6}\right)^{-1 / 3} .
$$

We now employ modular equations in $x$ and $y$ to calculate further values of $\lambda_{n}$.

\section{Theorem 5.1.}

$$
\begin{aligned}
& 32\left\{\left(\lambda_{n} \lambda_{289 n}\right)^{2 / 3}+\left(\lambda_{n} \lambda_{289 n}\right)^{-2 / 3}\right\}+80\left\{\left(\lambda_{n} \lambda_{289 n}\right)^{1 / 3}+\left(\lambda_{n} \lambda_{289 n}\right)^{-1 / 3}\right\} \\
& +118+\left(\lambda_{n}-\lambda_{n}^{-1}\right)^{1 / 3}\left(\lambda_{289 n}-\lambda_{289 n}^{-1}\right)^{1 / 3}\left(\left(\lambda_{n} \lambda_{289 n}\right)^{2 / 3}+\left(\lambda_{n} \lambda_{289 n}\right)^{-2 / 3}\right. \\
& \left.+35\left\{\left(\lambda_{n} \lambda_{289 n}\right)^{1 / 3}+\left(\lambda_{n} \lambda_{289 n}\right)^{-1 / 3}\right\}+56\right) \\
& -\left(\lambda_{n}-\lambda_{n}^{-1}\right)^{2 / 3}\left(\lambda_{289 n}-\lambda_{289 n}^{-1}\right)^{2 / 3} \\
& \cdot\left(\left(\lambda_{n} \lambda_{289 n}\right)^{1 / 3}+\left(\lambda_{n} \lambda_{289 n}\right)^{-1 / 3}-14\right)=\frac{1}{3}\left\{\left(\frac{\lambda_{n}}{\lambda_{289 n}}\right)+\left(\frac{\lambda_{n}}{\lambda_{289 n}}\right)^{-1}\right\} .
\end{aligned}
$$

Proof. The modular equation of degree 17 with which we start was proved by Chan and W.-C. Liaw [15] and is given by

$$
\begin{aligned}
& x^{6}+96 x^{5} y-240 x^{4} y^{2}+354 x^{3} y^{3}-240 x^{2} y^{4}+96 x y^{5}+y^{6} \\
& -3 x^{4}+105 x^{3} y-168 x^{2} y^{2}+105 x y^{3}-3 y^{4}+3 x^{2}+42 x y+3 y^{2}-1=0,
\end{aligned}
$$

where $x$ and $y$ are defined by (5.4) and (5.5) with $p=17$. Dividing both sides of (5.15) by $3 x^{3} y^{3}$, we obtain

$$
\begin{aligned}
& 32\left(\frac{x^{2}}{y^{2}}+\frac{y^{2}}{x^{2}}\right)-80\left(\frac{x}{y}+\frac{y}{x}\right)+118 \\
& -\frac{1}{x y}\left\{\left(\frac{x^{2}}{y^{2}}+\frac{y^{2}}{x^{2}}\right)-35\left(\frac{x}{y}+\frac{y}{x}\right)+56\right\} \\
& +\frac{1}{x^{2} y^{2}}\left\{\left(\frac{x}{y}+\frac{y}{x}\right)+14\right\}=\frac{1}{3}\left\{\frac{1}{x^{3} y^{3}}-\left(\frac{x^{3}}{y^{3}}+\frac{y^{3}}{x^{3}}\right)\right\},
\end{aligned}
$$

after a slight rearrangement. 
By (5.13), (5.14), and (5.16),

$$
\begin{aligned}
& 32\left\{\left(T U_{17}\right)^{-4}+\left(T U_{17}\right)^{4}\right\}-80\left\{\left(T U_{17}\right)^{-2}+\left(T U_{17}\right)^{2}\right\}+118 \\
& -\left(T^{6}+T^{-6}\right)^{1 / 3}\left(U_{17}^{6}+U_{17}^{-6}\right)^{1 / 3}\left(\left(T U_{17}\right)^{-4}+\left(T U_{17}\right)^{4}-35\right. \\
& \left.\cdot\left\{\left(T U_{17}\right)^{-2}+\left(T U_{17}\right)^{2}\right\}+56\right) \\
& +\left(T^{6}+T^{-6}\right)^{2 / 3}\left(U_{17}^{6}+U_{17}^{-6}\right)^{2 / 3}\left(\left(T U_{17}\right)^{-2}+\left(T U_{17}\right)^{2}+14\right) \\
& =\frac{1}{3}\left\{\left(\frac{T}{U_{17}}\right)^{6}+\left(\frac{T}{U_{17}}\right)^{-6}\right\} .
\end{aligned}
$$

Replacing $q$ by $-q$ in (5.17), setting $q=e^{-\pi \sqrt{n / 3}}$, and using (1.4), we complete the proof.

\section{Corollary 5.2.}

$$
\lambda_{289}=\left(\frac{\sqrt{34+13 \sqrt[3]{17}+5 \sqrt[3]{17^{2}}}}{2}+\frac{\sqrt{30+13 \sqrt[3]{17}+5 \sqrt[3]{17^{2}}}}{2}\right)^{6} .
$$

Proof. Set $n=1$ and $\lambda=\lambda_{289}$. It follows from Theorem 5.1 that

$$
118+32\left(\lambda^{2 / 3}+\lambda^{-2 / 3}\right)+80\left(\lambda^{1 / 3}+\lambda^{-1 / 3}\right)=\frac{\lambda+\lambda^{-1}}{3} .
$$

Let $\Lambda:=\lambda^{1 / 3}+\lambda^{-1 / 3}$. Then from (5.18), we have

$$
118+32\left(\Lambda^{2}-2\right)+80 \Lambda=\frac{\Lambda^{3}-3 \Lambda}{3} .
$$

Hence $\Lambda$ is a root of the equation

$$
\Lambda^{3}-96 \Lambda^{2}-243 \Lambda-162=0 .
$$

The proper solution for $\Lambda$ is

$$
\Lambda=32+13 \sqrt[3]{17}+5 \sqrt[3]{17^{2}} .
$$

Corollary 5.2 now follows from (4.2) and (4.4).

\section{Corollary 5.3.}

$$
\lambda_{17}=4+\sqrt{17} .
$$

Proof. Let $n=1 / 17$ in Theorem 5.1. Then by Lemma 4.9, we have

$$
171-64\left(\lambda_{17}-\lambda_{17}^{-1}\right)^{2 / 3}+6\left(\lambda_{17}-\lambda_{17}^{-1}\right)^{4 / 3}=\frac{1}{6}\left(\lambda_{17}^{2}+\lambda_{17}^{-2}\right) .
$$

We complete the proof by solving this equation for $\lambda_{17}$. 


\section{Theorem 5.4.}

$$
\lambda_{169}=\left(\frac{2+\sqrt{13}}{2}+\frac{\sqrt{13+4 \sqrt{13}}}{2}\right)^{6} .
$$

Proof. Let $x$ and $y$ be defined by (5.4) and (5.5) with $p=13$. In [20], W.-C. Liaw established a modular equation of degree 13, which, by (5.13) and (5.14), can be put in the abbreviated form

$$
\begin{aligned}
& \left\{\left(T U_{13}\right)^{-42}+\left(T U_{13}\right)^{42}\right\}+76142\left\{\left(T U_{13}\right)^{-36}+\left(T U_{13}\right)^{36}\right\} \\
& +1932468187\left\{\left(T U_{13}\right)^{-30}+\left(T U_{13}\right)^{30}\right\}+16346295812652 \\
& \times\left\{\left(T U_{13}\right)^{-24}+\left(T U_{13}\right)^{24}\right\}-42859027901079\left\{\left(T U_{13}\right)^{-18}\right. \\
& \left.+\left(T U_{13}\right)^{18}\right\}+30681672585330\left\{\left(T U_{13}\right)^{-12}+\left(T U_{13}\right)^{12}\right\} \\
& +44443969755835\left\{\left(T U_{13}\right)^{-6}+\left(T U_{13}\right)^{6}\right\}-90882188302360 \\
& +R(x, y)=0
\end{aligned}
$$

where $R(x, y)$ contains a factor $1 /\left(x^{3} y^{3}\right)$. Let $q=e^{-\pi / \sqrt{3}}$, recall that $\lambda_{1}=1$, and set $\lambda=\lambda_{169}$. Replacing $q$ by $-q$ in (5.19), by (1.4), we find that

$$
\begin{aligned}
& -\left(\lambda^{7}+\lambda^{-7}\right)+76142\left(\lambda^{6}+\lambda^{-6}\right)-1932468187\left(\lambda^{5}+\lambda^{-5}\right) \\
& +16346295812652\left(\lambda^{4}+\lambda^{-4}\right)+42859027901079\left(\lambda^{3}+\lambda^{-3}\right) \\
& +30681672585330\left(\lambda^{2}+\lambda^{-2}\right)-44443969755835\left(\lambda+\lambda^{-1}\right) \\
& -90882188302360=0,
\end{aligned}
$$

since $R(x, y)$ equals 0 after $q$ is replaced by $-q$, for

$$
1 / x^{3} y^{3}=\left(T^{6}+T^{-6}\right)\left(U_{13}^{6}+U_{13}^{-6}\right)
$$

is a factor of $R(x, y)$, and

$$
\begin{aligned}
& \left\{T^{6}(-q)+T^{-6}(-q)\right\}\left\{U_{13}^{6}(-q)+U_{13}^{-6}(-q)\right\} \\
& =-\left\{\left(\lambda_{1}-\lambda_{1}^{-1}\right)\left(\lambda_{169}-\lambda_{169}^{-1}\right)\right\} .
\end{aligned}
$$

Set $\Lambda=\lambda+\lambda^{-1}$. Then (5.20) has the equivalent form

$$
\begin{aligned}
& -\left(\Lambda^{7}-7 \Lambda^{5}+14 \Lambda^{3}-7 \Lambda\right)+76142\left(\Lambda^{6}-6 \Lambda^{4}+9 \Lambda^{2}-2\right) \\
& -1932468187\left(\Lambda^{5}-5 \Lambda^{3}+5 \Lambda\right)+16346295812652\left(\Lambda^{4}-4 \Lambda^{2}+2\right) \\
& +42859027901079\left(\Lambda^{3}-3 \Lambda\right)+30681672585330\left(\Lambda^{2}-2\right) \\
& -44443969755835 \Lambda-90882188302360=0,
\end{aligned}
$$

which simplifies to

$$
(\Lambda-2)\left(\Lambda^{2}-25380 \Lambda-39100\right)^{3}=0 .
$$

Therefore,

$$
\lambda+\lambda^{-1}=\Lambda=10(1269+352 \sqrt{13}) .
$$


Solving the quadratic equation above, we find that

$$
\lambda_{169}=6345+1760 \sqrt{13}+12 \sqrt{559221+155100 \sqrt{13}} .
$$

Using the formula [7, Eq. (3.1)]

$$
\left\{\left(32 b^{3}-6 b\right)+\sqrt{\left(32 b^{3}-6 b\right)^{2}-1}\right\}^{1 / 6}=\sqrt{b+\frac{1}{2}}+\sqrt{b-\frac{1}{2}},
$$

with $b=\frac{15+4 \sqrt{13}}{4}$, we deduce the value of $\lambda_{169}$.

Equation (5.19) can also be used to deduce $\lambda_{13}$.

\section{Theorem 5.5.}

$$
\begin{aligned}
\lambda_{361} & =\frac{1}{3}\left(2928581+1097504(19)^{1 / 3}+411296(19)^{2 / 3}\right. \\
& \left.+4 \sqrt{1608109304409+602648894772(19)^{1 / 3}+225846395748(19)^{2 / 3}}\right) .
\end{aligned}
$$

Proof. The proof is similar to that for Theorem 5.4. Let $x$ and $y$ be defined by (5.4) and (5.5), respectively, and set $u=x^{3}, v=y^{3}$, and $p=19$. Liaw [20] found a modular equation of degree 19, which we give in the abbreviated form,

$$
\begin{aligned}
& \left(\frac{u^{10}}{v^{10}}\right)+\left(\frac{v^{10}}{u^{10}}\right)-17571484\left\{\left(\frac{u^{9}}{v^{9}}\right)+\left(\frac{v^{9}}{u^{9}}\right)\right\}+102919027240030 \\
& \cdot\left\{\left(\frac{u^{8}}{v^{8}}\right)+\left(\frac{v^{8}}{u^{8}}\right)\right\}-200937885610911191740\left\{\left(\frac{u^{7}}{v^{7}}\right)+\left(\frac{v^{7}}{u^{7}}\right)\right\} \\
& +363165905126589014509\left\{\left(\frac{u^{6}}{v^{6}}\right)+\left(\frac{v^{6}}{u^{6}}\right)\right\}-2745050674147219542832 \\
& \cdot\left\{\left(\frac{u^{5}}{v^{5}}\right)+\left(\frac{v^{5}}{u^{5}}\right)\right\}+1669253999271588508904\left\{\left(\frac{u^{4}}{v^{4}}\right)+\left(\frac{v^{4}}{u^{4}}\right)\right\} \\
& -9487507697742191502320\left\{\left(\frac{u^{3}}{v^{3}}\right)+\left(\frac{v^{3}}{u^{3}}\right)\right\}-7070474114231105014510 \\
& \cdot\left\{\left(\frac{u^{2}}{v^{2}}\right)+\left(\frac{v^{2}}{u^{2}}\right)\right\}-7249503742499660191624\left\{\left(\frac{u}{v}\right)+\left(\frac{v}{u}\right)\right\} \\
& -29289891786172199497868+R(u, v)=0,
\end{aligned}
$$

where $R(u, v)$ is a sum of terms with a factor $1 /(u v)$, which equals 0 after setting $q=e^{-\pi / \sqrt{3}}$ and replacing $q$ by $-q$. With $\Lambda=\lambda_{361}+\lambda_{361}^{-1}$, we 
eventually find that

$$
\begin{aligned}
& \left(\Lambda^{10}-10 \Lambda^{8}+35 \Lambda^{6}-50 \Lambda^{4}+25 \Lambda^{2}-2\right) \\
& -17571484\left(\Lambda^{9}-9 \Lambda^{7}+27 \Lambda^{5}-30 \lambda^{3}+9 \Lambda\right) \\
& +102919027240030\left(\Lambda^{8}-8 \Lambda^{6}+20 \Lambda^{4}-16 \Lambda^{2}+2\right) \\
& -200937885610911191740\left(\Lambda^{7}-7 \Lambda^{5}+14 \lambda^{3}-7 \Lambda\right) \\
& +363165905126589014509\left(\Lambda^{6}-6 \Lambda^{4}+9 \Lambda^{2}-2\right) \\
& -2745050674147219542832\left(\Lambda^{5}-5 \Lambda^{3}+5 \Lambda\right) \\
& +1669253999271588508904\left(\Lambda^{4}-4 \Lambda^{2}+2\right) \\
& -9487507697742191502320\left(\Lambda^{3}-3 \Lambda\right) \\
& -7070474114231105014510\left(\Lambda^{2}-2\right) \\
& -7249503742499660191624 \Lambda-29289891786172199497868=0
\end{aligned}
$$

which simplifies to

$$
(\Lambda+2)\left(-18438200+7433420 \Lambda-5857162 \Lambda^{2}+\Lambda^{3}\right)^{3}=0 .
$$

Hence

$$
\Lambda=\frac{1}{3}\left(5857162+2195008(19)^{1 / 3}+822592(19)^{2 / 3}\right) .
$$

Solving the equation $\Lambda=\lambda_{361}+\lambda_{361}^{-1}$ for $\lambda_{361}$, we complete the proof.

The modular equation of degree 19 given above can also be used to calculate $\lambda_{19}$; see also Corollary 6.5(iii).

\section{6. $\lambda_{n}$ and Kronecker's limit formula.}

Let $m>0$ be square-free and let $K=\mathbb{Q}(\sqrt{-m})$, the imaginary quadratic field with discriminant $d$, where

$$
d=\left\{\begin{aligned}
-4 m, & \text { if }-m \equiv 2,3 \quad(\bmod 4), \\
-m, & \text { if }-m \equiv 1 \quad(\bmod 4) .
\end{aligned}\right.
$$

Let $d=d_{1} d_{2}$, where $d_{1}>0$ and, for $i=1,2, d_{i} \equiv 0$ or $1(\bmod 4)$. If $\mathcal{P}$ denotes a prime ideal in $K$, then the Gauss genus character $\chi$ is defined by

$$
\chi(\mathcal{P})= \begin{cases}\left(\frac{d_{1}}{N(\mathcal{P})}\right), & \text { if } \quad N(\mathcal{P}) \chi d_{1}, \\ \left(\frac{d_{2}}{N(\mathcal{P})}\right), & \text { if } \quad N(\mathcal{P}) \mid d_{1},\end{cases}
$$

where $N(\mathcal{P})$ is the norm of the ideal $\mathcal{P}$ and $(\vdots)$ denotes the Kronecker symbol. 
Let

$$
\Omega= \begin{cases}\sqrt{-m}, & \text { if }-m \equiv 2,3 \quad(\bmod 4), \\ \frac{1+\sqrt{-m}}{2}, & \text { if } \quad-m \equiv 1 \quad(\bmod 4) .\end{cases}
$$

It is known [21] that each ideal class in the class group $C_{K}$ contains primitive ideals which are $\mathbb{Z}$-modules of the form $\mathcal{A}=[a, b+\Omega]$, where $a$ and $b$ are rational integers, $a>0, a|N(b+\Omega)| b \mid, \leq a / 2, a$ is the smallest positive integer in $\mathcal{A}$ and $N(\mathcal{A})=a$. Hence, Siegel's Theorem [27, p. 72], obtained from Kronecker's limit formula, can be stated as follows.

Theorem 6.1 (Siegel). Let $\chi$ be a genus character arising from the decomposition $d=d_{1} d_{2}$. Let $h_{i}$ be the class number of the field $\mathbb{Q}\left(\sqrt{d_{i}}\right)$, $\omega$ and $\omega_{2}$ be the numbers of roots of unity in $K$ and $\mathbb{Q}\left(\sqrt{d_{2}}\right)$, respectively, and $\epsilon_{1}$ be the fundamental unit of $\mathbb{Q}\left(\sqrt{d_{1}}\right)$. Let

$$
F(\mathcal{A})=\frac{|\eta(z)|^{2}}{\sqrt{a}}
$$

where $z=(b+\Omega) / a$ with $[a, b+\Omega] \in \mathcal{A}^{-1}$. Then

$$
\epsilon_{1}^{\omega h_{1} h_{2} / \omega_{2}}=\prod_{\mathcal{A} \in C_{K}} F(\mathcal{A})^{-\chi(\mathcal{A})}
$$

Ramanathan utilized Theorem 6.1 to compute $\lambda_{n}$ and $\mu_{n}[\mathbf{2 2}$, Theorem 4].

Theorem 6.2 (Ramanathan). Let $3 n$ be a positive square free integer and let $K=\mathbb{Q}(\sqrt{-3 n})$ be an imaginary quadratic field such that each genus contains only one ideal class. Then

$$
\prod_{\chi} \epsilon_{1}^{t_{\chi}}= \begin{cases}\lambda_{n}, & \text { if } n \equiv 1 \quad(\bmod 4), \\ \mu_{n}, & \text { if } n \equiv 2,3 \quad(\bmod 4),\end{cases}
$$

where

$$
t_{\chi}=\frac{6 \omega h_{1} h_{2}}{\omega_{2} h}
$$

$h, h_{1}, h_{2}$ are the class numbers of $K, \mathbb{Q}\left(\sqrt{d_{1}}\right)$, and $\mathbb{Q}\left(\sqrt{d_{2}}\right)$, respectively, $\omega$ and $\omega_{2}$ are the numbers of roots of unity in $K$ and $\mathbb{Q}\left(\sqrt{d_{2}}\right)$, respectively, $\epsilon_{1}$ is the fundamental unit in $\mathbb{Q}\left(\sqrt{d_{1}}\right)$, and $\chi$ runs through all genus characters such that if $\chi$ corresponds to the decomposition $d_{1} d_{2}$, then either $\left(\frac{d_{1}}{3}\right)$ or $\left(\frac{d_{2}}{3}\right)=-1$ and therefore $d_{1}, d_{2}, h_{1}, h_{2}, \omega_{2}$, and $\epsilon_{1}$ are dependent on $\chi$.

By Theorem 6.2, 14 values of $\lambda_{n}$ and 19 of $\mu_{n}$ can be evaluated. Among them, Ramanujan recorded only the values of $\lambda_{n}$ for which the exponent $t_{\chi}=1$. We state all 14 values of such $\lambda_{n}$ 's in the following corollary. 


\section{Corollary 6.3.}

$$
\begin{aligned}
& \lambda_{5}=\frac{1+\sqrt{5}}{2}, \quad \lambda_{17}=4+\sqrt{17}, \\
& \lambda_{41}=32+5 \sqrt{41}, \quad \lambda_{65}=(8+\sqrt{65})\left(\frac{1+\sqrt{5}}{2}\right)^{6}, \\
& \lambda_{89}=500+53 \sqrt{89}, \quad \lambda_{145}=\left(\frac{1+\sqrt{5}}{2}\right)^{9}\left(\frac{5+\sqrt{29}}{2}\right)^{3}, \\
& \lambda_{161}=(16 \sqrt{23}+29 \sqrt{7})\left(\frac{3 \sqrt{3}+\sqrt{23}}{2}\right)^{3}, \\
& \lambda_{185}=(68+5 \sqrt{185})\left(\frac{1+\sqrt{5}}{2}\right)^{12}, \\
& \lambda_{209}=(46 \sqrt{11}+35 \sqrt{19})(2 \sqrt{3}+\sqrt{11})^{3}, \\
& \lambda_{265}=\left(\frac{7+\sqrt{53}}{2}\right)^{3}\left(\frac{1+\sqrt{5}}{2}\right)^{15}, \\
& \lambda_{385}=\left(\frac{1+\sqrt{5}}{2}\right)^{9}\left(\frac{5+\sqrt{21}}{2}\right)^{3}\left(\frac{\sqrt{7}+\sqrt{11}}{2}\right)^{3}\left(\frac{\sqrt{15}+\sqrt{11}}{2}\right)^{3}, \\
& \lambda_{1105}=(4+\sqrt{17})^{3}(8+\sqrt{65})^{3}\left(\frac{1+\sqrt{5}}{2}\right)^{12}\left(\frac{15+\sqrt{221}}{2}\right)^{3} \cdot \\
& \lambda_{665}=(14 \sqrt{35}+19 \sqrt{19})(2 \sqrt{5}+\sqrt{15}+\sqrt{19})^{3} \\
& \lambda_{1001}
\end{aligned}
$$

We next derive a theorem from Theorem 6.1 by which we can evaluate some $\lambda_{n}$ for $n \equiv 3(\bmod 4)$.

Theorem 6.4. Let $n>3$ be a positive square free integer, not divisible by 3 , and $n \equiv 3(\bmod 4)$. Let $K=\mathbb{Q}(\sqrt{-3 n})$ be an imaginary quadratic field such that each genus contains only one ideal class. Let $C_{0}$ be the principal ideal class containing $[1, \Omega]$, where $\Omega$ is defined by $(6.3)$, and $C_{1}$ and $C_{2}$ be nonprincipal ideal classes containing $[2,1+\Omega]$ and $[6,3+\Omega]$, respectively. 
Then

$$
\lambda_{n}=\left(\prod_{\chi\left(C_{1}\right)=-1} \epsilon_{1}^{t_{\chi}}\right)^{-1}\left(\prod_{\chi\left(C_{2}\right)=-1} \epsilon_{1}^{t_{\chi}}\right),
$$

where $t_{\chi}, d_{1}, d_{2}, h_{1}, h_{2}, \omega_{2}$, and $\epsilon_{1}$ are defined in Theorem 6.2 , and the products are over all characters $\chi$ (the first with $\chi\left(C_{1}\right)=-1$ and the second with $\left.\chi\left(C_{2}\right)=-1\right)$, associated with the decomposition $d=d_{1} d_{2}$, and therefore $d_{1}, d_{2}, h_{1}, h_{2}, \omega_{2}$, and $\epsilon_{1}$ are dependent on $\chi$.

Proof. If $\mathcal{A} \in C$ is any of the ideals $[1, \Omega],[2,1+\Omega]$, and $[6,3+\Omega]$, then $\mathcal{A} \sim \mathcal{A}^{-1}, C=C^{-1}$, and $\mathcal{A} \in C^{-1}$. For any ideal class $C$, not $C_{0}$ nor $C_{1}$, Ramanathan $[\mathbf{2 2}]$ showed that

$$
\sum_{\chi\left(C_{1}\right)=-1} \chi(C)=0
$$

which implies that

$$
\prod_{\chi\left(C_{1}\right)=-1} F(C)^{-\chi(C)}=1,
$$

where $F(C)$ is defined by (6.4). Therefore, by (6.5),

$$
\begin{aligned}
\prod_{\chi\left(C_{1}\right)=-1} \epsilon_{1}^{\omega h_{1} h_{2} / \omega_{2}} & =\prod_{\chi\left(C_{1}\right)=-1} \prod_{\mathcal{A} \in C_{0} \cup C_{1}} F(\mathcal{A})^{-\chi(\mathcal{A})} \\
& =\prod_{\mathcal{A} \in C_{0} \cup C_{1}} F(\mathcal{A})^{-\chi(\mathcal{A}) h / 2}=\left(\frac{F\left(C_{1}\right)}{F\left(C_{0}\right)}\right)^{h / 2},
\end{aligned}
$$

since the number of genus characters is $h$, and so the number of genus characters such that $\chi\left(C_{1}\right)=-1$ is $h / 2$. With a similar argument, we find that

$$
\prod_{\chi\left(C_{2}\right)=-1} \epsilon_{1}^{\omega h_{1} h_{2} / \omega_{2}}=\left(\frac{F\left(C_{2}\right)}{F\left(C_{0}\right)}\right)^{h / 2} .
$$

Dividing (6.7) into (6.8), we have

$$
\left(\frac{F\left(C_{2}\right)}{F\left(C_{1}\right)}\right)^{h / 2}=\frac{\prod_{\chi\left(C_{2}\right)=-1} \epsilon_{1}^{\omega h_{1} h_{2} / \omega_{2}}}{\prod_{\chi\left(C_{1}\right)=-1} \epsilon_{1}^{\omega h_{1} h_{2} / \omega_{2}}} .
$$

But, by (6.4),

$$
\frac{F\left(C_{2}\right)}{F\left(C_{1}\right)}=\frac{1}{\sqrt{3}}\left(\frac{\eta\left(\frac{1+i \sqrt{n / 3}}{2}\right)}{\eta\left(\frac{1+i \sqrt{3 n}}{2}\right)}\right)^{2} .
$$

The theorem now follows from (6.9), (6.10), and (1.4). 
We can apply Theorem 6.4 to evaluate $\lambda_{n}$ when $n=7,11,19,31,35,55$, $59,91,115,119,455$. We, first, determine all the values of such $\lambda_{n}$ when $\mathbb{Q}(\sqrt{-3 n})$ has class number 4 and each genus contains one ideal class.

Observe that $\chi\left(C_{1}\right)=-1$, where $\chi$ has the decomposition $d=d_{1} d_{2}$ such that $\left(\frac{d_{1}}{2}\right)=-1$ or $\left(\frac{d_{2}}{2}\right)=-1$, i.e., $d_{1} \equiv \pm 3$ or $d_{2} \equiv \pm 3(\bmod 8)$, and $\chi\left(C_{2}\right)=-1$, where $\left(\frac{d_{1}}{6}\right)=-1$ or $\left(\frac{d_{2}}{6}\right)=-1$.

\section{Corollary 6.5.}

$$
\begin{aligned}
& \lambda_{7}=(2+\sqrt{3})^{3 / 2}\left(\frac{\sqrt{3}+\sqrt{7}}{2}\right)^{-3 / 2}, \\
& \lambda_{11}=(2 \sqrt{3}+\sqrt{11})^{3 / 2}(10+3 \sqrt{11})^{-1 / 2}, \\
& \lambda_{19}=(151+20 \sqrt{57})^{3 / 4}(2+\sqrt{3})^{-3 / 2}, \\
& \lambda_{31}=(2+\sqrt{3})^{9 / 2}\left(\frac{2 \sqrt{7}+\sqrt{31}}{2}\right)^{-3 / 2}, \\
& \lambda_{59}=(102 \sqrt{3}+23 \sqrt{59})^{3 / 2}(530+69 \sqrt{59})^{-1 / 2} .
\end{aligned}
$$

Proof. For all five values of $n$ above, the corresponding imaginary quadratic field $\mathbb{Q}(\sqrt{-3 n})$ has class number 4 and genus number 4 . Since the values of $\lambda_{7}$ and $\lambda_{11}$ are already determined in Corollaries 4.11 and 4.12, respectively, we will discuss only the last three values.

The following tables summarize the needed information about ideal classes and their characters.

Let $K=\mathbb{Q}(\sqrt{-57})$.

\begin{tabular}{|l|c|c|c|rc|c|c|c|c|}
\hline$d_{1}$ & $d_{2}$ & $\chi$ & $C$ & $\begin{array}{r}\chi\left(C_{0}\right) \\
\chi\left(C_{2}\right)\end{array}\left(C_{1}\right)$ & $h_{1}$ & $h_{2}$ & $\omega_{2}$ & $\epsilon_{1}$ \\
\hline 1 & -228 & $\chi_{0}$ & {$[1, \Omega]$} & $\begin{array}{r}1 \\
1\end{array}$ & 1 & & & & \\
\hline 57 & -4 & $\chi_{1}$ & {$[2,1+\Omega]$} & $\begin{array}{r}1 \\
-1\end{array}$ & -1 & 1 & 1 & 4 & $151+20 \sqrt{57}$ \\
\hline 44 & -3 & $\chi_{2}$ & {$[6,3+\Omega]$} & $\begin{array}{r}1 \\
-1\end{array}$ & -1 & 1 & 1 & 6 & \\
\hline 12 & -11 & $\chi_{3}$ & {$[3, \Omega]$} & $\begin{array}{r}1 \\
1\end{array}$ & -1 & 1 & 1 & 2 & $2+\sqrt{3}$ \\
\hline
\end{tabular}

By Theorem 6.4,

$$
\lambda_{19}=\frac{\prod_{\chi_{1}, \chi_{2}} \epsilon_{1}^{t_{\chi}}}{\prod_{\chi_{2}, \chi_{3}} \epsilon_{1}^{t_{\chi}}}=\frac{\epsilon_{1}^{t_{\chi_{1}}}}{\epsilon_{1}^{t_{\chi_{3}}}}=(151+20 \sqrt{57})^{3 / 4}(2+\sqrt{3})^{-3 / 2} .
$$

Let $K=\mathbb{Q}(\sqrt{-93})$. 


\begin{tabular}{|c|c|c|c|c|c|c|c|c|}
\hline$d_{1}$ & $d_{2}$ & $\chi$ & $C$ & $\begin{array}{l}\chi\left(C_{0}\right) \chi\left(C_{1}\right) \\
\chi\left(C_{2}\right) \chi\left(C_{3}\right)\end{array}$ & $h_{1}$ & $h_{2}$ & $\omega_{2}$ & $\epsilon_{1}$ \\
\hline 1 & -372 & $\chi_{0}$ & {$[1, \Omega]$} & $\begin{array}{ll}1 & 1 \\
1 & 1\end{array}$ & & & & \\
\hline 93 & -4 & $\chi_{1}$ & {$[2,1+\Omega]$} & $\begin{array}{ll}1 & -1 \\
1 & -1\end{array}$ & 1 & 1 & 4 & $\frac{29+3 \sqrt{93}}{2}$ \\
\hline 44 & -3 & $\chi_{2}$ & {$[6,3+\Omega]$} & $\begin{array}{rr}1 & -1 \\
-1 & 1\end{array}$ & 1 & 1 & 6 & \\
\hline 12 & -11 & $\chi_{3}$ & {$[3, \Omega]$} & $\begin{array}{rr}1 & 1 \\
-1 & -1\end{array}$ & 1 & 3 & 2 & $2+\sqrt{3}$ \\
\hline
\end{tabular}

By Theorem 6.4,

$$
\lambda_{31}=\frac{\prod_{\chi_{2}, \chi_{3}} \epsilon_{1}^{t_{\chi}}}{\prod_{\chi_{1}, \chi_{2}} \epsilon_{1}^{t_{\chi}}}=\frac{\epsilon_{1}^{t_{\chi_{3}}}}{\epsilon_{1}^{t_{\chi_{1}}}}=(2+\sqrt{3})^{9 / 2}\left(\frac{29+3 \sqrt{93}}{2}\right)^{-3 / 4} .
$$

Let $K=\mathbb{Q}(\sqrt{-177})$.

\begin{tabular}{|c|c|c|c|c|c|c|c|c|}
\hline$d_{1}$ & $d_{2}$ & $\chi$ & $C$ & $\begin{array}{l}\chi\left(C_{0}\right) \chi\left(C_{1}\right) \\
\chi\left(C_{2}\right) \chi\left(C_{3}\right)\end{array}$ & $h_{1}$ & $h_{2}$ & $\omega_{2}$ & $\epsilon_{1}$ \\
\hline 1 & -708 & $\chi_{0}$ & {$[1, \Omega]$} & $\begin{array}{ll}1 & 1 \\
1 & 1\end{array}$ & & & & \\
\hline 177 & -4 & $\chi_{1}$ & {$[2,1+\Omega]$} & $\begin{array}{rr}1 & 1 \\
-1 & -1\end{array}$ & 1 & 1 & 4 & $62423+4692 \sqrt{177}$ \\
\hline 236 & -3 & $\chi_{2}$ & {$[6,3+\Omega]$} & $\begin{array}{ll}1 & -1 \\
1 & -1\end{array}$ & 1 & 1 & 6 & $530+69 \sqrt{59}$ \\
\hline 12 & -59 & $\chi_{3}$ & {$[3, \Omega]$} & $\begin{array}{rr}1 & -1 \\
-1 & 1\end{array}$ & 1 & 3 & 2 & \\
\hline
\end{tabular}

By Theorem 6.4,

$$
\begin{aligned}
\lambda_{59}=\frac{\prod_{\chi_{1}, \chi_{3}} \epsilon_{1}^{t_{\chi}}}{\prod_{\chi_{2}, \chi_{3}} \epsilon_{1}^{t_{\chi}}}=\frac{\epsilon_{1}^{t_{\chi_{1}}}}{\epsilon_{1}^{t_{\chi_{2}}}} & =(62423+4692 \sqrt{177})^{3 / 4}(530+69 \sqrt{59})^{-1 / 2} \\
& =(102 \sqrt{3}+23 \sqrt{59})^{3 / 2}(530+69 \sqrt{59})^{-1 / 2} .
\end{aligned}
$$

Next, we give examples of evaluations of $\lambda_{n}$ when $\mathbb{Q}(\sqrt{-3 n})$ has class number 8 and each genus contains one ideal class.

Corollary 6.6.

(i)

$$
\lambda_{35}=(\sqrt{21}+2 \sqrt{5})^{3 / 2}(4+\sqrt{15})^{3 / 2}(2+\sqrt{5})^{-1}\left(\frac{\sqrt{5}+\sqrt{7}}{\sqrt{2}}\right)^{-1},
$$


(ii)

$$
\lambda_{55}=(10+3 \sqrt{11})^{3 / 2}(2+\sqrt{3})^{3}(2+\sqrt{5})^{-1}\left(\frac{13+\sqrt{165}}{2}\right)^{-3 / 4},
$$

(iii)

$$
\lambda_{91}=(25+4 \sqrt{39})^{3 / 2}(727+44 \sqrt{273})^{3 / 4}(2+\sqrt{3})^{-3 / 2}\left(\frac{5+\sqrt{21}}{2}\right)^{-3 / 2},
$$

$$
\lambda_{115}=(24+5 \sqrt{23})^{3 / 2}(26 \sqrt{5}+7 \sqrt{69})^{3 / 2}(2+\sqrt{5})^{-2}(2+\sqrt{3})^{-3 / 2} \text {, }
$$

$$
\lambda_{119}=(4+\sqrt{17})^{3}(50+7 \sqrt{51})^{3 / 2}(120+11 \sqrt{119})^{-1 / 2}\left(\frac{19+\sqrt{357}}{2}\right)^{-3 / 4} .
$$

Proof. We give the calculation for only (i), as the calculations in the remaining four cases are similar. Let $K=\mathbb{Q}(\sqrt{-105})$. Then its discriminant $d=-420$.

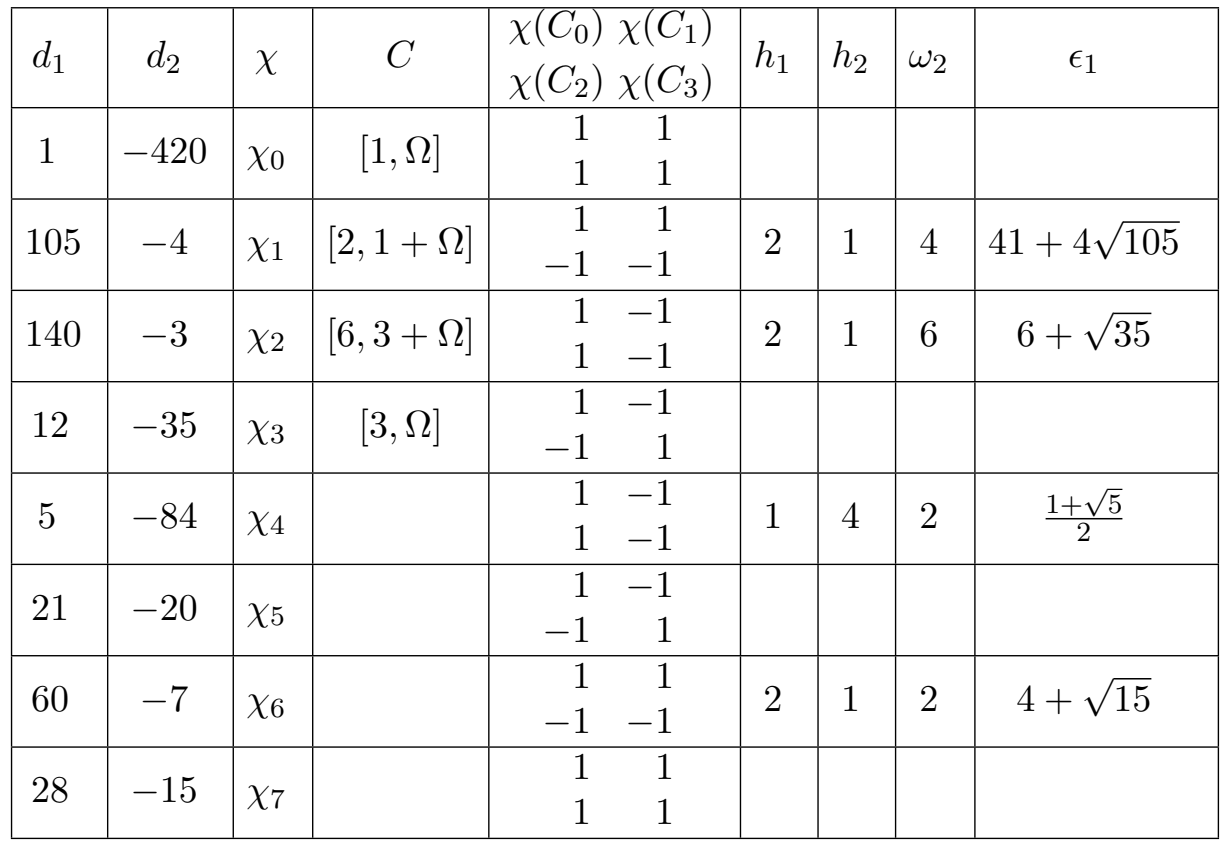

By Theorem 6.4, 


$$
\begin{aligned}
\lambda_{35} & =\frac{\prod_{\chi_{1}, \chi_{3}, \chi_{5}, \chi_{6}} \epsilon_{1}^{t_{\chi}}}{\prod_{\chi_{2}, \chi_{3}, \chi_{4}, \chi_{5}} \epsilon_{1}^{t_{\chi}}}=\frac{\epsilon_{1}^{t_{\chi_{1}}} \epsilon_{1}^{t_{\chi_{6}}}}{\epsilon_{1}^{t_{\chi_{2}}} \epsilon_{1}^{t_{\chi_{4}}}} \\
& =(41+4 \sqrt{105})^{3 / 4}(4+\sqrt{15})^{3 / 2}(6+\sqrt{35})^{-1 / 2}\left(\frac{1+\sqrt{5}}{2}\right)^{-3} .
\end{aligned}
$$

Lastly, by a similar calculation, we can determine $\lambda_{455} ;$ here $\mathbb{Q}(\sqrt{-1365})$ has class number 16, and each genus contains one ideal class.

\section{Corollary 6.7.}

$$
\begin{aligned}
\lambda_{455} & =(14+\sqrt{195})^{3 / 2}(8+\sqrt{65})^{3}(6+\sqrt{35})^{3}(41+4 \sqrt{105})^{3 / 2} \\
& \times(2+\sqrt{5})^{-1}(4+\sqrt{15})^{-3 / 2}(64+3 \sqrt{455})^{-1 / 2}\left(\frac{37+\sqrt{1365}}{2}\right)^{-3 / 4} .
\end{aligned}
$$

\section{An empirical process for computing $\lambda_{n}$.}

In this section, we describe an empirical process which can be used to derive values of $\lambda_{n}$ for $n=73,97,193,217$, and 241. This empirical process is analogous to those used by G.N. Watson [29], [30] in his computations of the Ramanujan-Weber class invariants $G_{n}$ and $g_{n}$.

Theorem 7.1. We have

$$
\begin{aligned}
& \lambda_{73}=\left(\sqrt{\frac{11+\sqrt{73}}{8}}+\sqrt{\frac{3+\sqrt{73}}{8}}\right)^{6}, \\
& \lambda_{97}=\left(\sqrt{\frac{17+\sqrt{97}}{8}}+\sqrt{\frac{9+\sqrt{97}}{8}}\right)^{6},
\end{aligned}
$$

$$
\lambda_{241}=(16+\sqrt{241}+\sqrt{496+32 \sqrt{241}})^{3},
$$

$$
\lambda_{217}=\left(\sqrt{\frac{1901+129 \sqrt{217}}{8}}+\sqrt{\frac{1893+129 \sqrt{217}}{8}}\right)^{3 / 2}
$$

$$
\cdot\left(\sqrt{\frac{1597+108 \sqrt{217}}{4}}+\sqrt{\frac{1593+108 \sqrt{217}}{4}}\right)^{3 / 2},
$$

(v) $\quad \lambda_{193}^{1 / 3}+\frac{1}{\lambda_{193}^{1 / 3}}=\frac{1}{4}(39+3 \sqrt{193}+\sqrt{2690+194 \sqrt{193}})$. 
Proof. By computing the numerical value of $\lambda_{73}$ using (1.1), we assume that

$$
\lambda_{73}^{1 / 3}+\frac{1}{\lambda_{73}^{1 / 3}}=7.77200187 \ldots=\frac{7+\sqrt{73}}{2} .
$$

Solving this quadratic equation, we arrive at

$$
\lambda_{73}=\left(\frac{7+\sqrt{73}}{4}+\sqrt{\frac{53+7 \sqrt{73}}{8}}\right)^{3} .
$$

Verifying that the value above is the same as Ramanujan's value for $\lambda_{73}$ is straightforward.

The computations of $\lambda_{97}$ and $\lambda_{241}$ are similar. We assume that

$$
\lambda_{97}^{1 / 3}+\frac{1}{\lambda_{97}^{1 / 3}}=11.42442890089 \ldots=\frac{13+\sqrt{97}}{2}
$$

and

which yield

$$
\lambda_{241}^{1 / 3}+\frac{1}{\lambda_{241}^{1 / 3}}=63.04834939 \ldots=32+2 \sqrt{241},
$$

$$
\lambda_{97}=\left(\frac{13+\sqrt{97}}{4}+\sqrt{\frac{125+13 \sqrt{97}}{8}}\right)^{3}
$$

and

$$
\lambda_{241}=(16+\sqrt{241}+\sqrt{496+32 \sqrt{241}})^{3} .
$$

The computation of $\lambda_{217}$ is different from those of the previous three values. We compute $\lambda_{217}$ and $\lambda_{31 / 7}$ numerically and assume that

$$
\left(\lambda_{217} \lambda_{31 / 7}\right)^{1 / 3}+\frac{1}{\left(\lambda_{217} \lambda_{31 / 7}\right)^{1 / 3}}=56.4618397 \ldots=27+2 \sqrt{217}
$$

and

$$
\left(\frac{\lambda_{217}}{\lambda_{31 / 7}}\right)^{1 / 3}+\left(\frac{\lambda_{31 / 7}}{\lambda_{217}}\right)^{1 / 3}=43.5963797 \ldots=\frac{43+3 \sqrt{217}}{2},
$$

which imply that

$$
\begin{aligned}
\lambda_{217}= & \left(\sqrt{\frac{1901+129 \sqrt{217}}{8}}+\sqrt{\frac{1893+129 \sqrt{217}}{8}}\right)^{3 / 2} \\
& \times\left(\sqrt{\frac{1597+108 \sqrt{217}}{4}}+\sqrt{\frac{1593+108 \sqrt{217}}{4}}\right)^{3 / 2} .
\end{aligned}
$$


In [14], this empirical method will be put on a firm foundation, and it will be shown that $\lambda_{n}$ can be explicitly determined when $n \equiv 1(\bmod 4)$, $3 \nmid n$ and the class group of $\mathbb{Q}(\sqrt{-3 n})$ is of the type $\mathbb{Z}_{2} \oplus \mathbb{Z}_{2} \oplus \cdots \oplus \mathbb{Z}_{2} \oplus \mathbb{Z}_{4}$. This result is analogous to that associated with the Ramanujan-Weber class invariant $G_{n}$ proved in [13]. It is clear that this method works for groups of the type $\mathbb{Z}_{2} \oplus \mathbb{Z}_{2} \oplus \cdots \oplus \mathbb{Z}_{2}$ (which is the class group of imaginary quadratic fields having one class per genus). Hence, the empirical process illustrated here can also be used to determine the values of $\lambda_{n}$ given in Section 6 .

It remains to determine the value of $\lambda_{193}$. The class group of $\mathbb{Q}(\sqrt{-579})$ is $\mathbb{Z}_{8}$, and so it does not belong to the set of $n$ 's that we discuss here. However, we know that $[\mathbf{1 4}] t:=\lambda_{193}+\lambda_{193}^{-1}$ lies in a quartic extension of $\mathbb{Q}(\sqrt{-579})$ and so we may use MAPLE V's "minpoly" function to guess a minimal polynomial of degree 4 satisfied by $t$. Our final result is

$$
\lambda_{193}^{1 / 3}+\frac{1}{\lambda_{193}^{1 / 3}}=\frac{1}{4}(39+3 \sqrt{193}+\sqrt{2690+194 \sqrt{193}}) .
$$

A rigorous proof of (7.1) can be found in [14].

\section{Evaluation of the modular $j$-invariant.}

The base $q$ in the alternative cubic theory is defined by

$$
q:=q_{3}:=\exp \left(-\frac{2 \pi}{\sqrt{3}} \frac{{ }_{2} F_{1}\left(\frac{1}{3}, \frac{2}{3} ; 1 ; 1-\alpha\right)}{{ }_{2} F_{1}\left(\frac{1}{3}, \frac{2}{3} ; 1 ; \alpha\right)}\right), \quad 0<\alpha<1,
$$

where ${ }_{2} F_{1}(a, b ; c ; z)$ is defined by (1.5). Recall the fundamental inversion formula for the cubic theta functions [4, Lemma 2.9],

$$
\alpha:=\alpha(q)=\frac{c^{3}(q)}{a^{3}(q)},
$$

where the cubic theta functions $a(q), b(q)$, and $c(q)$ are defined by (5.1)(5.3). By (5.6)-(5.8), we find that

$$
\frac{1}{\alpha(q)}=\frac{f^{12}(-q)}{27 q f^{12}\left(-q^{3}\right)}+1 .
$$

Let

$$
s:=s(q):=\frac{f^{12}(-q)}{27 q f^{12}\left(-q^{3}\right)} .
$$

From the relation between the $j$-invariant and the modulus $\sqrt{\alpha}$ in the cubic theory [5]

$$
j(3 \tau)=27 \frac{(1+8(1-\alpha))^{3}}{(1-\alpha) \alpha^{3}}, \quad q=e^{2 \pi i \tau},
$$


and (8.3), we deduce that

$$
j(3 \tau)=27(9 s+1)^{3} \frac{s+1}{s} .
$$

Thus by (2.3),

$$
\gamma_{2}(3 \tau)=\sqrt[3]{j(3 \tau)}=3(9 s+1) \sqrt[3]{\frac{s+1}{s}} .
$$

Let $\tau=(3+\sqrt{-n}) / 6$. Then by (2.4), (1.3), and (1.4), we obtain the following theorem after replacing $n$ by $3 n$ and dividing both sides by -1 .

\section{Theorem 8.1.}

$$
32 J_{3 n}=-\gamma_{2}\left(\frac{3+\sqrt{-3 n}}{2}\right)=3\left(9 \lambda_{n}^{2}-1\right) \sqrt[3]{\frac{\lambda_{n}^{2}-1}{\lambda_{n}^{2}}} .
$$

By Theorem 8.1, we can determine the corresponding values of $J_{n}$ for known values of $\lambda_{n}$. For example, the values of $J_{n}$ in the table (2.5) are easy consequences of Theorem 8.1. We give another example in the corollary below.

\section{Corollary 8.2.}

$$
J_{123}=15(8+\sqrt{41}) \sqrt[3]{32+5 \sqrt{41}} .
$$

Proof. Let $n=41$ in Theorem 8.1 and use the value of $\lambda_{41}$ in Table (1.2).

\section{9. $\lambda_{n}$ is a unit when $n$ is odd and not divisible by 3 .}

Recall that an order $\mathcal{O}_{f}$ with conductor $f$ in a quadratic field $K$ is a subset $\mathcal{O}_{f} \subset K$ such that

(i) $\mathcal{O}_{f}$ is a subring of $K$ containing 1 ,

(ii) $\mathcal{O}_{f}$ is a finitely generated $\mathbb{Z}$-module,

(iii) $\mathcal{O}_{f}$ contains a $\mathbb{Q}$-basis of $K$, and

(iv) $\left[\mathcal{O}_{K}: \mathcal{O}_{f}\right]=f$,

where $\mathcal{O}_{K}=\mathcal{O}_{1}$ is the ring of integers of $K$.

If $\alpha_{1}$ and $\alpha_{2}$ generate an $\mathcal{O}_{f}$-ideal $\mathcal{A}_{\mathcal{O}_{f}}$ over $\mathbb{Z}$, we say that $\left[\alpha_{1}, \alpha_{2}\right]$ is a basis of $\mathcal{A}_{\mathcal{O}_{f}}$. An $\mathcal{O}_{K}$ ideal $\mathcal{A}_{\mathcal{O}_{f}}$ is said to be proper if

$$
\mathcal{O}_{f}=\left\{\beta \in K: \beta \mathcal{A}_{\mathcal{O}_{f}} \subset \mathcal{A}_{\mathcal{O}_{f}}\right\} .
$$

Every ideal in $\mathcal{O}_{K}$ prime to $f$ is proper.

If $\nu$ is an algebraic integer and $\mathcal{A}$ is an $\mathcal{O}_{K}$ ideal, we write $\nu \approx \mathcal{A}$ to mean that $\nu \mathcal{O}_{L}=\mathcal{A} \mathcal{O}_{L}$ in some larger number field $L$. Similarly, if $\nu_{1}$ and $\nu_{2}$ are algebraic integers, we write $\nu_{1} \approx \nu_{2}$ to mean that $\nu_{1} / \nu_{2}$ is a unit. 
Theorem 9.1 (Deuring, [19, p. 43]). Let $f$ be a positive integer and $p^{t} \| f$, where $p$ is a prime and $t$ is a nonnegative integer. Let $a, b, c$, and $d$ be integers, $M:=\left(\begin{array}{ll}a & b \\ c & d\end{array}\right)$ be a matrix with determinant $p$, and $\Delta(\tau)=q(q ; q)_{\infty}^{24}$, where $q=e^{2 \pi i \tau}$, with $\operatorname{Im} \tau>0$. Define

$$
\Phi_{M}(\tau)=p^{12} \frac{\Delta\left(M\left(\begin{array}{l}
\tau_{1} \\
\tau_{2}
\end{array}\right)\right)}{\Delta\left(\begin{array}{l}
\tau_{1} \\
\tau_{2}
\end{array}\right)},
$$

where $\tau=\tau_{1} / \tau_{2}$ and $\Delta\left(\begin{array}{c}\tau_{1} \\ \tau_{2}\end{array}\right):=\Delta\left(\left(\begin{array}{c}\tau_{1} \\ \tau_{2}\end{array}\right)\right):=\tau_{2}^{-12} \Delta(\tau)$. Let $\left[\alpha_{1}, \alpha_{2}\right]$ be a basis of a proper $\mathcal{O}_{f}$-ideal $\mathcal{A}_{O_{f}}$, and set $\alpha=\alpha_{1} / \alpha_{2}$. The action of $M$ on the basis $\left[\alpha_{1}, \alpha_{2}\right]$ is defined as $M\left[\alpha_{1}, \alpha_{2}\right]=\left[a \alpha_{1}+b \alpha_{2}, c \alpha_{1}+d \alpha_{2}\right]$.

I. When $p$ splits completely in $K$, namely $p=\mathcal{P} \overline{\mathcal{P}}$, then

(I.a) $\Phi_{M}(\alpha)$ is a unit if $M\left[\alpha_{1}, \alpha_{2}\right]$ is a basis of a proper $\mathcal{O}_{f p}$-ideal,

(I.b) $\Phi_{M}(\alpha) \approx p^{12}$ if $M\left[\alpha_{1}, \alpha_{2}\right]$ is a basis of a proper $\mathcal{O}_{f p^{-1}}$-ideal,

(I.c) if $p \nmid$ f, then $\Phi_{M}(\alpha) \approx \overline{\mathcal{P}}^{12}$ and $\Phi_{M}(\alpha) \approx \mathcal{P}^{12}$ when $M\left[\alpha_{1}, \alpha_{2}\right]$ is a basis of $\mathcal{A}_{\mathcal{O}_{f}} \mathcal{P}_{\mathcal{O}_{f}}$ and $M\left[\alpha_{1}, \alpha_{2}\right]$ is a basis of $\mathcal{A}_{\mathcal{O}_{f}} \overline{\mathcal{P}}_{\mathcal{O}_{f}}$, respectively.

II. When $p$ ramifies in $K$, namely $p=\mathcal{P}^{2}$, then

(II.a) $\Phi_{M}(\alpha) \approx p^{6 / p^{t+1}}$ if $M\left[\alpha_{1}, \alpha_{2}\right]$ is a basis of a proper $\mathcal{O}_{f p}$-ideal,

(II.b) $\Phi_{M}(\alpha) \approx p^{12-6 / p^{t}}$ if $M\left[\alpha_{1}, \alpha_{2}\right]$ is a basis of a proper $\mathcal{O}_{f p^{-1}}$-ideal,

(II.c) $\Phi_{M}(\alpha) \approx p^{6}$ if $M\left[\alpha_{1}, \alpha_{2}\right]$ is a basis of $\mathcal{A}_{\mathcal{O}_{f}} \mathcal{P}_{\mathcal{O}_{f}}$.

III. When $p$ is inert in $K$, then

(III.a) $\Phi_{M}(\alpha) \approx p^{12 / p^{t}(p+1)}$ if $M\left[\alpha_{1}, \alpha_{2}\right]$ is a basis of a proper $\mathcal{O}_{f p}$-ideal, (III.b) $\Phi_{M}(\alpha) \approx p^{12\left(1-1 / p^{t-1}(p+1)\right)}$ if $M\left[\alpha_{1}, \alpha_{2}\right]$ is a basis of a proper $\mathcal{O}_{f p^{-1}}$ ideal.

Corollary 9.2. $\lambda_{n}$ is a unit if $n$ is odd and not divisible by 3.

Proof. Let $n=f^{2} d$, where $d$ is square-free, and let $K=\mathbb{Q}(\sqrt{-3 d})$.

(i) First, suppose that $n \equiv 1(\bmod 4)$. Then $d \equiv 1(\bmod 4)$, and the ring of integers $\mathcal{O}_{K}=\left[\frac{1+\sqrt{-3 d}}{2}, 1\right]$, and $(3)=\mathcal{P}^{2}$ ramifies in $\mathcal{O}_{K}$. Consider the order $\mathcal{O}_{f}$ of conductor $f$. Then $\mathcal{O}_{f}$ has a basis $\mathcal{O}_{f}=\left[\frac{f+\sqrt{-3 f^{2} d}}{2}, 1\right]$. Let $M=\left(\begin{array}{ll}1 & f \\ 0 & 3\end{array}\right)$. Then $M\left[\frac{f+\sqrt{-3 f^{2} d}}{2}, 1\right]=\left[\frac{3 f+\sqrt{-3 f^{2} d}}{2}, 3\right]$ is a basis of $\mathcal{P} \mathcal{O}_{f}$. Hence by (II.c) in Theorem 9.1, we have

$$
\Phi_{M}\left(\frac{f+\sqrt{-3 f^{2} d}}{2}\right) \approx 3^{6} .
$$


On the other hand,

$$
\begin{aligned}
& \Phi_{M}\left(\frac{f+\sqrt{-3 f^{2} d}}{2}\right)=3^{12} \frac{\Delta\left(\left(\begin{array}{ll}
1 & f \\
0 & 3
\end{array}\right)\left(\begin{array}{c}
\left.f+\sqrt{-3 f^{2} d}\right) \\
2
\end{array}\right)\right.}{\Delta\left(^{f+\sqrt{-3 f^{2} d}}\right)} \\
& =3^{12} \frac{\Delta\left(\begin{array}{l}
\left.3 f+\sqrt{-3 f^{2} d}\right) \\
6
\end{array}\right)}{\Delta\left(\begin{array}{c}
f+\sqrt{-3 f^{2} d} \\
2
\end{array}\right)}=3^{12} \frac{6^{-12} \Delta\left(\frac{3 f+\sqrt{-3 f^{2} d}}{6}\right)}{2^{-12} \Delta\left(\frac{f+\sqrt{-3 f^{2} d}}{2}\right)} \\
& =\frac{\Delta\left(\frac{f+\sqrt{-f^{2} d / 3}}{2}\right)}{\Delta\left(\frac{f+\sqrt{-3 f^{2} d}}{2}\right)}=\frac{\Delta\left(\frac{1+\sqrt{-n / 3}}{2}\right)}{\Delta\left(\frac{1+\sqrt{-3 n}}{2}\right)},
\end{aligned}
$$

where the last equality follows from the facts that $\Delta(\tau+1)=\Delta(\tau)$ and $f$ is odd. But, since $\Delta(\tau)=\eta^{24}(\tau)$ by (1.3),

$$
\Phi_{M}\left(\frac{f+\sqrt{-3 f^{2} d}}{2}\right)=\left(\frac{\eta\left(\frac{1+i \sqrt{n / 3}}{2}\right)}{\eta\left(\frac{1+i \sqrt{3 n}}{2}\right)}\right)^{24} .
$$

Thus by (1.4),

$$
\Phi_{M}\left(\frac{f+\sqrt{-3 f^{2} d}}{2}\right)=\left(3 \sqrt{3} \lambda_{n}\right)^{4}=3^{6} \lambda_{n}^{4} .
$$

Combining (9.2) and (9.3), we have $3^{6} \approx 3^{6} \lambda_{n}^{4}$. Hence $\lambda_{n}^{4}$ is a unit, and so is $\lambda_{n}$.

(ii) Suppose that $n \equiv 3(\bmod 4)$. Then $d \equiv 3(\bmod 4), \mathcal{O}_{K}=[\sqrt{-3 d}, 1]$, and $(3)=\mathcal{P}^{2}$ ramifies in $\mathcal{O}_{K}$. The order $\mathcal{O}_{f}$ of conductor $f$ has a basis $\mathcal{O}_{f}=\left[\sqrt{-3 f^{2} d}, 1\right]$. Let $\left[f+\sqrt{-3 f^{2} d}, 2\right]$ be a basis of a proper $\mathcal{O}_{f}$-ideal $\mathcal{A}_{\mathcal{O}_{f}}$. Let $M=\left(\begin{array}{ll}1 & f \\ 0 & 3\end{array}\right)$. Since $M\left[f+\sqrt{-3 f^{2} d}, 2\right]=\left[3 f+\sqrt{-3 f^{2} d}, 6\right]$ is a basis of $\mathcal{A}_{\mathcal{O}_{f}} \mathcal{P}_{\mathcal{O}_{f}}$, we deduce from (II.c) in Theorem 9.1 that

$$
\Phi_{M}\left(\frac{f+\sqrt{-3 f^{2} d}}{2}\right) \approx 3^{6} .
$$

As we have shown above,

$$
\Phi_{M}\left(\frac{f+\sqrt{-3 f^{2} d}}{2}\right)=3^{6} \lambda_{n}^{4} .
$$

Hence we can conclude that $\lambda_{n}$ is a unit. 
Corollary 9.3. If $n$ is odd and congruent to 2 modulo 3 , then $3^{-3 / 2} \lambda_{3 n}$ is a unit.

Proof. Let $n=f^{2} d$, where $d$ is square-free, and let $K=\mathbb{Q}(\sqrt{-d})$.

(i) Suppose that $3 n \equiv 1(\bmod 4)$. Then $d \equiv 3(\bmod 4), \mathcal{O}_{K}=\left[\frac{1+\sqrt{-d}}{2}, 1\right]$, and $(3)=\mathcal{P} \overline{\mathcal{P}}$ splits completely in $\mathcal{O}_{K}$, because $-d \equiv 1(\bmod 3)$. Let $\mathcal{O}_{3 f}$ be an order of $\mathcal{O}_{K}$ of conductor $3 f$. Then $\mathcal{O}_{3 f}=\left[\frac{3 f+3 \sqrt{-f^{2} d}}{2}, 1\right]$. Let $M=\left(\begin{array}{ll}1 & 0 \\ 0 & 3\end{array}\right)$. Then $M\left[\frac{3 f+3 \sqrt{-f^{2} d}}{2}, 1\right]=\left[\frac{3 f+3 \sqrt{-f^{2} d}}{2}, 3\right]$ is a basis of $3 \mathcal{O}_{f}$. Hence, by (I.b) of Theorem 9.1,

$$
\Phi_{M}\left(\frac{3 f+3 \sqrt{-f^{2} d}}{2}\right) \approx 3^{12} .
$$

On the other hand, by a calculation like that in the previous proof,

$$
\begin{aligned}
\Phi_{M}\left(\frac{3 f+3 \sqrt{-f^{2} d}}{2}\right) & =3^{12} \frac{\Delta\left(\left(\begin{array}{ll}
1 & 0 \\
0 & 3
\end{array}\right)\left(\begin{array}{c}
\left.3 f+3 \sqrt{-f^{2} d}\right) \\
2
\end{array}\right)\right.}{\Delta\left(\begin{array}{c}
3 f+3 \sqrt{-f^{2} d} \\
2
\end{array}\right)} \\
& =\left(3 \sqrt{3} \lambda_{3 n}\right)^{4}=3^{6} \lambda_{3 n}^{4},
\end{aligned}
$$

by (1.3) and (1.4). Combining (9.4) and (9.5), we deduce that $3^{-3 / 2} \lambda_{3 n}$ is a unit.

(ii) Suppose that $3 n \equiv 3(\bmod 4)$. Then $d \equiv 1(\bmod 4), \mathcal{O}_{K}=[\sqrt{-d}, 1]$, and $(3)=\mathcal{P} \overline{\mathcal{P}}$ splits completely in $\mathcal{O}_{K}$. Let $\mathcal{O}_{3 f}=\left[3 \sqrt{-f^{2} d}, 1\right]$ be an order of $\mathcal{O}_{K}$ with conductor $3 f$. Let $\left[f+3 \sqrt{-f^{2} d}, 2\right]$ be a basis of a proper $\mathcal{O}_{3 f^{-}}$ ideal $\mathcal{A}_{3 f}$. Let $M=\left(\begin{array}{ll}1 & f \\ 0 & 3\end{array}\right)$. Then $M\left[f+3 \sqrt{-f^{2} d}, 2\right]=\left[3 f+3 \sqrt{-f^{2} d}, 6\right]$ is a basis of $3 \mathcal{B}_{\mathcal{O}_{f}}$, where $\mathcal{B}_{\mathcal{O}_{f}}=\left[f+\sqrt{-f^{2} d}, 2\right]$ is a proper ideal of $\mathcal{O}_{f}$. Hence by (I.b) in Theorem 9.1,

$$
\Phi_{M}\left(\frac{f+3 \sqrt{-f^{2} d}}{2}\right) \approx 3^{12} .
$$

On the other hand, by calculations like those above,

$$
\Phi_{M}\left(\frac{f+3 \sqrt{-f^{2} d}}{2}\right)=3^{12} \frac{\Delta\left(\left(\begin{array}{cc}
1 & f \\
0 & 3
\end{array}\right)\left(\begin{array}{l}
\left.f+3 \sqrt{-f^{2} d}\right) \\
2
\end{array}\right)\right.}{\Delta\left(\begin{array}{l}
f+3 \sqrt{-f^{2} d} \\
2
\end{array}\right)}=3^{6} \lambda_{3 n}^{4}
$$

where the last equality was deduced from (1.3) and (1.4). Hence, from (9.6) and (9.7), we can conclude that $3^{-3 / 2} \lambda_{3 n}$ is a unit. 
Corollary 9.4. If $n$ is odd and congruent to 1 modulo 3 , then $3^{-3 / 4} \lambda_{3 n}$ is a unit.

Proof. Let $n=f^{2} d$, where $d$ is square-free, and let $K=\mathbb{Q}(\sqrt{-d})$.

(i) Suppose that $3 n \equiv 1(\bmod 4)$. Then $d \equiv 3(\bmod 4), \mathcal{O}_{k}=\left[\frac{1+\sqrt{-d}}{2}, 1\right]$, and 3 is inert in $\mathcal{O}_{K}$, because $-d \equiv 2(\bmod 3)$. By an argument similar to that in (i) in the proof of Corollary 9.3 and by the use of (III.b) in Theorem 9.1, we can deduce that

$$
3^{6} \lambda_{3 n}^{4}=\Phi_{M}\left(\frac{3 f+3 \sqrt{-f^{2} d}}{2}\right) \approx 3^{12(1-1 / 4)}=3^{9} .
$$

Hence $3^{-3 / 4} \lambda_{3 n}$ is a unit.

(ii) We can deduce the same result when $3 n \equiv 3(\bmod 4)$ by using a method similar to that in (ii) in the proof of Corollary 9.3 and by using (III.b) of Theorem 9.1.

Corollary 9.5. If $n=3^{2 t}$ for $t \geq 1$, then $3^{-\frac{3}{2}\left(1-3^{-t}\right)} \lambda_{n}$ is a unit.

Proof. Suppose that $n \equiv 1(\bmod 4)$. Let $K=\mathbb{Q}(\sqrt{-3})$. Then $\mathcal{O}_{K}=$ $\left[\frac{1+\sqrt{-3}}{2}, 1\right]$ and $\left[\frac{3^{t}+\sqrt{-3^{2 t+1}}}{2}, 1\right]$ is a basis of the order $\mathcal{O}_{3^{t}}$ with conductor $3^{t}$. Note that $(3)=\mathcal{P}^{2}$ ramifies in $\mathcal{O}_{K}$. Let $M=\left(\begin{array}{ll}1 & 0 \\ 0 & 3\end{array}\right)$. Then $M\left[\frac{3^{t}+\sqrt{-3^{2 t+1}}}{2}, 1\right]$ $=\left[\frac{3^{t}+\sqrt{-3^{2 t+1}}}{2}, 3\right]$ is a basis of the principal ideal $3 \mathcal{O}_{3^{t-1}}$. By (II.b) in Theorem 9.1 ,

$$
\Phi_{M}\left(\frac{3^{t}+\sqrt{-3^{2 t+1}}}{2}\right) \approx 3^{12-6 \cdot 3^{-t}} .
$$

But, since, by (1.3) and (1.4),

$$
\Phi_{M}\left(\frac{3^{t}+\sqrt{-3^{2 t+1}}}{2}\right)=3^{6} \lambda_{n}^{4}
$$

$3^{-\frac{3}{2}\left(1-3^{-t}\right)} \lambda_{n}$ is a unit.

We are very grateful to W.-C. Liaw for providing the necessary modular equations of degrees 13 and 19. We also thank Jinhee Yi for discovering several calculational errors in an earlier draft of this paper.

\section{References}

[1] B.C. Berndt, Ramanujan's Notebooks, Part III, Springer-Verlag, New York, 1991, MR 92j:01069, Zbl 733.11001.

[2] _ Ramanujan's Notebooks, Part IV, Springer-Verlag, New York, 1994, MR 95e:11028, Zbl 785.11001. 
[3] _ Ramanujan's Notebooks, Part V, Springer-Verlag, New York, 1998, MR 99f:11024, Zbl 886.11001.

[4] B.C. Berndt, S. Bhargava and F.G. Garvan, Ramanujan's theories of elliptic functions to alternative bases, Trans. Amer. Math. Soc., 347 (1995), 4163-4244, MR 97h:33034, Zbl 843.33012.

[5] B.C. Berndt and H.H. Chan, Ramanujan and the modular $j$-invariant, Canad. Math. Bull., 42 (1999), 427-440, CMP 1727340.

[6] _ Eisenstein series and approximations to $\pi$, Illinois J. Math., 45 (2001), 75-90.

[7] B.C. Berndt, H.H. Chan and L.-C. Zhang, Radicals and units in Ramanujan's work, Acta Arith., 87 (1998), 145-158, MR 99m:11125, Zbl 919.11069.

[8] J.M. Borwein and P.B. Borwein, Pi and the AGM, Wiley, New York, 1987, MR 89a:11134, Zbl 611.10001.

[9] _ More Ramanujan-type series for $1 / \pi$, in 'Ramanujan Revisited', eds. G.E. Andrews, R.A. Askey, B.C. Berndt, K.G. Ramanathan and R.A. Rankin, Academic Press, Boston, (1988), 359-374, MR 89d:11118, Zbl 652.10019.

[10] A cubic counterpart of Jacobi's identity and the AGM, Trans. Amer. Math. Soc., 323 (1991), 691-701, MR 91e:33012, Zbl 725.33014.

[11] _ Class number three Ramanujan type series for $1 / \pi$, J. Comput. Appl. Math., 46 (1993), 281-290, MR 94h:11113, Zbl 818.65010.

[12] H.H. Chan, On Ramanujan's cubic transformation formula for ${ }_{2} F_{1}(1 / 3,2 / 3 ; 1 ; z)$, Math. Proc. Cambridge Philos. Soc., 124 (1998), 193-204, MR 99f:11054, Zbl 949.33002.

[13] _ Ramanujan-Weber class invariant $G_{n}$ and Watson's empirical process, J. London Math. Soc., 57 (1998), 545-561, MR 99j:11134, Zbl 922.11117.

[14] H.H. Chan, A. Gee and V. Tan, Cubic singular moduli, Ramanujan's class invariants $\lambda_{n}$ and the explicit Shimura reciprocity law, Pacific J. Math., to appear.

[15] H.H. Chan and W.-C. Liaw, On Russell-type modular equations, Pacific J. Math., 192 (2000), 219-238.

[16] H.H. Chan, W.-C. Liaw and V. Tan, Ramanujan's class invariant $\lambda_{n}$ and a new class of series for $1 / \pi$, J. London Math. Soc. (2), 64 (2001), 93-106.

[17] K. Chandrasekharan, Elliptic Functions, Springer-Verlag, Berlin, 1985, MR 87e:11058, Zbl 575.33001.

[18] D.A. Cox, Primes of the Form $x^{2}+n y^{2}$, Wiley, New York, 1989, MR 90m:11016, Zbl 701.11001.

[19] M. Deuring, Die Klassenkörper der komplexes Multiplication, in 'Enz. Math. Wiss. Band $I_{2}$ ', Heft 10, Teil II, Stuttgart, 1958.

[20] W.-C. Liaw, Contributions to Ramanujan's Theory of Modular Equations in Classical and Alternative Bases, Ph.D. Thesis, University of Illinois, 1999.

[21] R. Mollin and L.-C. Zhang, Orders in quadratic fields, II, Proc. Japan Acad. Ser. A, 69 (1993), 368-371, MR 95d:11153, Zbl 809.11063.

[22] K.G. Ramanathan, Some applications of Kronecker's limit formula, J. Indian Math. Soc., 52 (1987), 71-89, MR 90j:11112, Zbl 682.12002.

[23] _ On some theorems stated by Ramanujan, in 'Number Theory and Related Topics', Tata Institute of Fundamental Research Studies in Mathematics, Oxford University Press, Bombay, (1989), 151-160, MR 98g:33027, Zbl 746.11009. 
[24] S. Ramanujan, Modular equations and approximations to $\pi$, Quart. J. Math. (Oxford), 45 (1914), 350-372.

[25] N Notebooks, (2 volumes), Tata Institute of Fundamental Research, Bombay, 1957, MR 20 \#6340, Zbl 138.24201.

[26] The Lost Notebook and Other Unpublished Papers, Narosa, New Delhi, 1988, Zbl 639.01023.

[27] C.L. Siegel, Advanced Analytic Number Theory, Tata Institute of Fundamental Research, Bombay, 1980, MR 83m:10001, Zbl 478.10001.

[28] H. Stark Values of L-functions at $s=1$ (i). L-functions for quadratic forms, Advances in Math., 7 (1971), 301-343, MR 44 \#6620, Zbl 263.10015.

[29] G.N. Watson, Theorems stated by Ramanujan (XIV): A singular modulus, J. London Math. Soc., 6 (1931), 126-132.

[30] _ Some singular moduli (I), Quart. J. Math., 3 (1932), 81-98.

[31] H. Weber, Lehrbuch der Algebra, dritter Band, Chelsea, New York, 1961.

Received February 11, 2000 and revised June 19, 2000.

Department of Mathematics

UNIVERSITY OF ILLINOIS

URBANA, IL 61801

E-mail address: berndt@math.uiuc.edu

Department of Mathematics

National University of Singapore

Kent Ridge, Singapore 119260

SINGAPORE

E-mail address: chanhh@math.nus.edu.sg

Department of Mathematics

The Ohio State University

Columbus, OH 43210

E-mail address: kang@math.ohio-state.edu

Department of Mathematics

Southwest Missouri State University

Springfield, Missouri 65804

E-mail address: liz917f@smsu.edu 